\title{
Molecular inner-shell photoabsorption/photoionization cross sections at core-valence- separated coupled cluster level: Theory and examples
}

Tenorio, Bruno Nunes Cabral; Moitra, Torsha; Nascimento, Marco Antonio Chaer; Rocha, Alexandre Braga; Coriani, Sonia

Published in:

Journal of Chemical Physics

Link to article, DOI:

$10.1063 / 1.5096777$

Publication date:

2019

Document Version

Publisher's PDF, also known as Version of record

Link back to DTU Orbit

Citation $(A P A)$ :

Tenorio, B. N. C., Moitra, T., Nascimento, M. A. C., Rocha, A. B., \& Coriani, S. (2019). Molecular inner-shell photoabsorption/photoionization cross sections at core-valence-separated coupled cluster level: Theory and examples. Journal of Chemical Physics, 150(22), [224104]. https://doi.org/10.1063/1.5096777

\section{General rights}

Copyright and moral rights for the publications made accessible in the public portal are retained by the authors and/or other copyright owners and it is a condition of accessing publications that users recognise and abide by the legal requirements associated with these rights.

- Users may download and print one copy of any publication from the public portal for the purpose of private study or research.

- You may not further distribute the material or use it for any profit-making activity or commercial gain

- You may freely distribute the URL identifying the publication in the public portal 


\section{Molecular inner-shell photoabsorption/ photoionization cross sections at core- valence-separated coupled cluster level: Theory and examples}

Cite as: J. Chem. Phys. 150, 224104 (2019); https://doi.org/10.1063/1.5096777

Submitted: 19 March 2019 . Accepted: 14 May 2019 . Published Online: 12 June 2019

Bruno Nunes Cabral Tenorio (D), Torsha Moitra (D), Marco Antonio Chaer Nascimento, Alexandre Braga Rocha (D), and Sonia Coriani (iD)
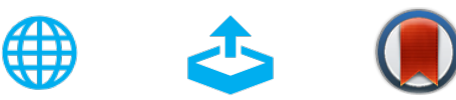

View Online

Export Citation

\section{ARTICLES YOU MAY BE INTERESTED IN}

Symplectic integration and physical interpretation of time-dependent coupled-cluster theory

The Journal of Chemical Physics 150, 144106 (2019); https://doi.org/10.1063/1.5085390

Theoretical study of the absolute inner-shell photoionization cross sections of the formic acid and some of its hydrogen-bonded clusters

The Journal of Chemical Physics 150, 154308 (2019); https://doi.org/10.1063/1.5088491

Generalized oscillator strengths of carbon disulfide calculated by multireference configuration interaction

The Journal of Chemical Physics 150, 174116 (2019); https://doi.org/10.1063/1.5090613

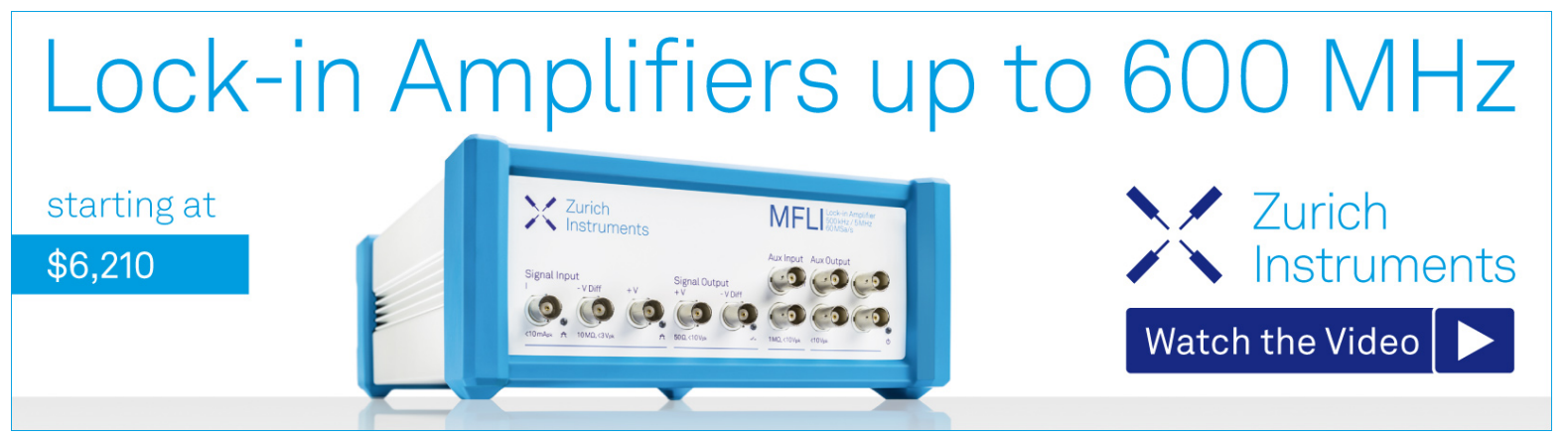




\title{
Molecular inner-shell photoabsorption/ photoionization cross sections at core-valence-separated coupled cluster level: Theory and examples
}

\author{
Cite as: J. Chem. Phys. 150, 224104 (2019); doi: 10.1063/1.5096777 \\ Submitted: 19 March 2019 - Accepted: 14 May 2019 • \\ Published Online: 12 June 2019
}
Bruno Nunes Cabral Tenorio,' (D) Torsha Moitra, ${ }^{2}$ (D) Marco Antonio Chaer Nascimento, Alexandre Braga Rocha, ${ }^{1, a)}$ (D) and Sonia Coriani ${ }^{2, b)}$

\begin{abstract}
AFFILIATIONS
${ }^{1}$ Instituto de Química, Universidade Federal do Rio de Janeiro, UFRJ, Av. Athos da Silveira Ramos, 149, Rio de Janeiro, RJ 21941-909, Brazil

${ }^{2}$ DTU Chemistry, Technical University of Denmark, Kemitorvet, Building 207, DK-2800 Kongens Lyngby, Denmark
\end{abstract}

\begin{abstract}
Note: This paper is part of the JCP special collection on Ultrafast Spectroscopy and Diffraction from XUV to X-ray.
a) Electronic mail: rocha@iq.ufrj.br

${ }^{b}$ Electronic mail: soco@kemi.dtu.dk
\end{abstract}

\begin{abstract}
Oxygen, nitrogen, and carbon K-shell photoabsorption and photoionization cross sections have been calculated within core-valence-separated coupled cluster (CC) linear response theory for a number of molecular systems, namely, water, ammonia, ethylene, carbon dioxide, acetaldehyde, furan, and pyrrole. The cross sections below and above the K-edge core ionization thresholds were obtained, on the same footing, from $L^{2}$ basis set calculations of the discrete electronic pseudospectrum yielded by an asymmetric-Lanczos-based formulation of CC linear response theory at the CC singles and doubles (CCSD) and CC singles and approximate doubles (CC2) levels. An analytic continuation procedure for both discrete and continuum cross sections as well as a Stieltjes imaging procedure for the photoionization cross section were applied and the results critically compared.
\end{abstract}

\section{Published under license by AIP Publishing. https://doi.org/10.1063/1.5096777}

\section{INTRODUCTION}

Processes involving the electron in the continuum, such as photoionization, play an important role in many of the modern atomic and molecular physical chemistry fields of knowledge. The interest in the photoionization processes of inner-shell electrons, for instance, has been growing with the advent of modern facilities exploiting X-ray radiation as the probing light. Near edge $\mathrm{X}$-ray absorption fine structure (NEXAFS) is one important type of X-ray absorption spectroscopy (XAS) with a large range of recent applications $^{1-16}$ that benefits from the development of theoretical approaches capable of yielding accurate spectra. Many quantum mechanical methods relying on quadratically integrable $\left(L^{2}\right)$ basis sets have been used in order to simulate the discrete region of NEXAFS electronic spectra, such as multiconfigurational wavefunction methods, ${ }^{17-19,21-25}$ Random Phase Approximation (RPA), ${ }^{26}$ the Static Exchange method (STEX), ${ }^{27,28}$ Time-Dependent Density Functional Theory (TDDFT), ${ }^{29-32}$ the Algebraic Diagrammatic Construction (ADC) approaches, ${ }^{33-38}$ and the Coupled Cluster (CC) ansatz. ${ }^{20,39-43}$ We also refer the interested reader to Ref. 44 for a recent overview.

Description of the spectral region above the ionization limit with $L^{2}$ methods requires, however, additional efforts, as the inclusion of an appropriate description of the electronic continuum is required. The Stieltjes imaging technique ${ }^{45,46}$ and the analytic continuation procedure based on the Padé approximants ${ }^{47-50}$ have been used since the 1970s to obtain photoionization cross sections with $L^{2}$ methods. The Stieltjes imaging technique achieved popularity at 
the end of the eighties, where it was applied primarily within the RPA for Hartree-Fock and multiconfigurational self-consistent field wavefunctions. ${ }^{25,51-53}$ More recently, Averbukh and co-workers proposed to apply Stieltjes Imaging to the (unconverged) block-Lanczos pseudospectra obtained within the second-order ADC(2) formalism to compute the converged ionization and autoionization cross sections in the vacuum ultraviolet (VUV)-UV frequency region. ${ }^{54,55} \mathrm{~A}$ similar approach has also been used on the Lanczos pseudospectra obtained from the hierarchy of coupled cluster approximations CC singles (CCS), CC approximate doubles (CC2), and CC singles and doubles (CCSD) $)^{56,57}$ to compute the photoionization and photodetachment cross sections of valence electrons of closed shell atoms, small molecules, and ions.

The analytic continuation procedure based on Padé approximants has been applied to the Lanczos CCSD pseudospectra to obtain both photoionization cross section in the VUV-UV frequency region and dynamic polarizabilities ${ }^{58}$ for a series of atoms and molecules and then further extended to the TDDFT approximation. ${ }^{59}$

Inner-shell photoionization cross section spectra relying on $L^{2}$ basis sets have also been recently obtained for a number of molecules by Neville et al. ${ }^{60}$ based on the application of Stieltjes imaging to $\mathrm{ADC}(2)-\mathrm{x}$ Lanczos pseudospectra of both ground and excited states, and by Tenorio et al. ${ }^{61}$ for ground-state only using the analytic continuation procedure on discretized representations of the bound and continuum states obtained from full-space Lanczos-based CC linear response $^{41}$ and from TDDFT. At the CC level, however, the investigation was limited to quite small systems since very large Lanczos chain lengths were required to access the core excited states. Herein, we investigate the cross sections below and above the K-edge core ionization thresholds obtained using a core-valence separated CC linear-response scheme based on the asymmetric Lanczos algorithm [herein labeled core-valence-separated (CVS)-CCLR], ${ }^{41,42}$ together with both an analytic continuation procedure for the discrete and continuum cross sections, and a Stieltjes imaging procedure ${ }^{46}$ for the photoionization cross section. The oxygen, nitrogen, and carbon K-edges of water $\left(\mathrm{H}_{2} \mathrm{O}\right)$, ammonia $\left(\mathrm{NH}_{3}\right)$, ethylene $\left(\mathrm{C}_{2} \mathrm{H}_{4}\right)$, carbon dioxide $\left(\mathrm{CO}_{2}\right)$, acetaldehyde $\left(\mathrm{CH}_{3} \mathrm{CHO}\right)$, furan $\left(\mathrm{C}_{4} \mathrm{H}_{4} \mathrm{O}\right)$, and pyrrole $\left(\mathrm{C}_{4} \mathrm{H}_{4} \mathrm{NH}\right)$ have been considered. This work also serves as a validation step toward the generalization of our methodologies to the determination of core photoionization cross sections of transient states, which are of interest for the simulation of timeresolved X-ray spectroscopy, in the spirit of the work presented by Neville et al.

\section{COMPUTATIONAL METHODOLOGIES}

\section{A. The CVS-CCLR Lanczos pseudospectrum}

The CC ansatz is defined by the exponential parametrization $|C C\rangle=\exp (T)|\mathrm{HF}\rangle$, where $|\mathrm{HF}\rangle$ is the reference (here HartreeFock) wavefunction, and the cluster operator $T$ is a sum of excitation operators $\tau_{\mu}$, each weighted by the corresponding amplitude $t_{\mu}$, $T=\sum_{\mu} t_{\mu} \tau_{\mu}$. The ground state energy and amplitudes are conventionally determined by projection of the Schrödinger equation onto the reference state and onto a manifold of excitations $|\mu\rangle$ out of the reference state, respectively,

$$
\begin{aligned}
E & =\langle\mathrm{HF}|\exp (-T) H \exp (T)| \mathrm{HF}\rangle ; \\
\Omega_{\mu} & =\langle\mu|\exp (-T) H \exp (T)| \mathrm{HF}\rangle=0 .
\end{aligned}
$$

Within CCLR theory, ${ }^{63,64}$ excitation energies $\left(\omega_{k}\right)$ and left $\left(L_{k}\right)$ and right $\left(R_{k}\right)$ excitation vectors can be obtained by solving the asymmetric eigenvalue equations

$$
\mathbf{A} R_{k}=\omega_{k} R_{k}, \quad L_{k} \mathbf{A}=\omega_{k} L_{k}
$$

under the biorthogonality condition $L_{j} R_{k}=\delta_{i k}$. The Jacobian matrix $\mathbf{A}$ is defined as

$$
A_{\mu \nu}=\frac{\partial \Omega_{\mu}}{\partial t_{v}}=\left\langle\mu\left|\exp (-T)\left[H, \tau_{v}\right] \exp (T)\right| \mathrm{HF}\right\rangle .
$$

One approach to solve Eq. (2) yielding a discretized spectrum covering the whole frequency range consists in building a truncated tridiagonal representation $\mathbf{T}$ of the Jacobian matrix $\mathbf{A}$ by application of an asymmetric Lanczos algorithm (or an Arnoldi algorithm), followed by its straightforward diagonalization. ${ }^{40,41}$ The nonzero elements of the tridiagonal matrix $\mathbf{T}=\mathbf{P}^{T} \mathbf{A} \mathbf{Q}$, where $\mathbf{P}^{T} \mathbf{Q}=\mathbf{1}$, are given by

$$
\begin{gathered}
T_{l l} \equiv \alpha_{l}=\mathbf{p}_{l}^{T} \mathbf{A} \mathbf{q}_{l}, \quad T_{l+1, l} \equiv \beta_{l}=\sqrt{\mathbf{p}_{l+l}^{T} \mathbf{q}_{l+1}} ; \\
T_{l, l+1} \equiv \gamma_{l}=\operatorname{sgn}\left\{\mathbf{p}_{l+1}^{T} \mathbf{q}_{l+1}\right\} \beta_{l}
\end{gathered}
$$

with

$$
\begin{aligned}
& \mathbf{q}_{l+1}=\beta_{l}^{-1}\left(\mathbf{A q}_{l}-\gamma_{l-1} \mathbf{q}_{l-1}-\alpha_{l} \mathbf{q}_{l}\right), \\
& \mathbf{p}_{l+1}^{T}=\gamma_{l}^{-1}\left(\mathbf{p}_{l}^{T} \mathbf{A}-\beta_{l-1} \mathbf{p}_{l-1}^{T}-\alpha_{l} \mathbf{p}_{l}^{T}\right),
\end{aligned}
$$

where $\mathbf{p}_{i}$ and $\mathbf{q}_{i}$ are columns of the $\mathbf{P}$ and $\mathbf{Q}$ matrices, respectively. Diagonalization of $\mathbf{T}$, conveniently truncated to a dimension (chain length) $J \ll n$, with $n$ being the full dimension, generates an effective excitation spectrum. The latter is known to converge from the bottom and from the top toward the exact excitation spectrum. $^{40,41}$

Choosing as Lanczos seeds the (bi-)normalized right-handside vectors of CCLR, ${ }^{41,65}$ that is, $\mathbf{q}_{1}=u_{X}^{-1} \boldsymbol{\xi}^{X}$ and $\mathbf{p}_{1}^{T}=v_{X}^{-1} \boldsymbol{\eta}^{X}$ (where $u_{X}=\left\|\xi^{X}\right\|$ and $v_{X}=u_{X}^{-1} \boldsymbol{\eta}^{X} \xi^{X}$ ), yields an approximate diagonal representation of the complex linear response function in terms of the eigenvectors of the Lanczos pseudospectrum. ${ }^{40,41}$ The residues of the linear response function give access to the transition strengths

$$
S_{0 \rightarrow j}^{X X}=u_{X} v_{X} L_{j 1}^{(J)} R_{1 j}^{(J)}-v_{X}^{2} \sum_{l} \mathcal{F}_{l j} \frac{L_{j 1} L_{l 1}}{\left(\omega_{j}+\omega_{l}\right)}
$$

for operator component $X$. See Refs. 40 and 41 for further definitions and details. When the operator $X$ is the electric dipole operator, the discrete dipole oscillator strengths $f_{j}$ for transitions from state 0 to state $j$ (in the length gauge) are obtained as

$$
f_{j}=\frac{2}{3} \omega_{j}\left(S_{0 \rightarrow j}^{X X}+S_{0 \rightarrow j}^{Y Y}+S_{0 \rightarrow j}^{Z Z}\right) .
$$

As the Lanczos algorithm is known to converge from the extremes, to obtain converged core excitation energies would require using a very large number of chain vectors, in other words, a very large matrix $\mathbf{T}$, which becomes quickly prohibitive even for medium sized 
systems. One can overcome this limitation by taking advantage of the almost nonexistent coupling between valence and core excited states and set elements corresponding to such couplings to zero during the iterative construction of the T matrix with negligible loss of accuracy within the chosen approximate level of theory. Via the CVS scheme, ${ }^{42,43,66}$ which is regarded as an efficient procedure to remove the continuum sea of valence ionizations from the inner-shell excitation region, the Lanczos approach yields straightforwardly a pseudospectrum starting at the lowest dipole-allowed core excitation of the chosen edge.

The CVS is easily introduced within the asymmetric Lanczos algorithm by applying, at each iteration, the projector

$$
\begin{cases}\mathcal{P}_{I}^{v} b_{i}^{a}=0, & \text { if } i \neq I, \\ \mathcal{P}_{I}^{v} b_{i j}^{a b}=0, & \text { if } i \neq I \text { or } j \neq I\end{cases}
$$

to both the $\mathbf{p}_{l}^{T}$ and $\mathbf{q}_{l}$ vectors and their linear transformations $\mathbf{p}_{l}^{T} \mathbf{A}$ and $\mathbf{A} \mathbf{q}_{l}$. The projector removes all vector elements not referencing at least one core orbital or a set of selected core orbitals $I$. In this way, the Lanczos trial-vector bases $\mathbf{P}^{T}$ and $\mathbf{Q}$ only contain excitations involving at least one core orbital, effectively decoupling them from excitations with contributions from occupied valence orbitals only. Diagonalization of the tridiagonal matrix yields the core excitations occurring as lowest roots, hereby quickly converging to the exact results with significantly smaller Lanczos chain lengths, and the Lanczos eigenvectors only refer to core excitations. The oscillator strengths and cross sections are computed directly from them, without further modifications to the general procedure.

\section{B. The Stieltjes imaging approach and the XAS cross section below and above the ionization limit}

The absorption cross section $\sigma(\omega)$ measures the probability for a photon of energy $\omega$ to be absorbed. It is proportional to the differential oscillator strength $f(\omega)$,

$$
\sigma(\omega)[\mathrm{Mb}]=2 \pi^{2} \alpha a_{0}^{2} \times 10^{18} f(\omega)
$$

where $\alpha$ is the fine structure constant, $a_{0}$ is the Bohr radius in centimeters, and $1 \mathrm{Mb}=10^{-18} \mathrm{~cm}^{2}$.

The XAS photoabsorption cross section can be partitioned into two contributions, ${ }^{60}$

$$
\sigma(\omega)=\sigma^{b}(\omega)+\sigma^{c}(\omega)
$$

where $\sigma^{b}(\omega)$ is the cross section arising from excitations into bound states and $\sigma^{c}(\omega)$ is the one arising from excitations into continuum states-that is, the photoionization cross section. Below the ionization threshold, the cross section $\sigma^{b}$ can be obtained by phenomenologically broadening the CC excitation energies $\hbar \omega_{j}$ and oscillator strengths $f_{j}$ obtained, as done here, via the asymmetric Lanczos procedure $^{40,41}$

$$
\sigma^{b}(\omega)=C \omega \sum_{j=1}^{N} \frac{3 f_{j}}{2 \omega_{j}} g\left(\omega, \omega_{j}\right),
$$

with $g\left(\omega, \omega_{j}\right)$ being the Lorentzian line shape function $g\left(\omega, \omega_{j}\right)=$ $\frac{\gamma}{\pi\left[\left(\omega-\omega_{j}\right)^{2}+\gamma^{2}\right]}$.
Above the ionization limit, however, the cross section cannot be determined directly from the discrete oscillator strengths obtained in the $L^{2}$ basis, due to the different normalizations of the "true" discrete states [below the ionization energies (IE)] and the "true" continuum states describing the outgoing electron. The former are normalized to a Kronecker- $\delta$, while the latter are normalized to a Dirac $\delta$-function. When dealing with photoionization, the discrete $f_{j}$ are replaced by a continuous oscillator strength function $f(\omega)$, which in the dipole-gauge is given by

$$
f(\omega)=\frac{2}{3}|\langle 0|\hat{\mu}| \phi(\omega)\rangle|^{2},
$$

where $\phi(\omega)$ is a continuum solution of the Schrödinger equation. The continuum and discrete wavefunctions satisfy different boundary conditions.

As suggested by Langhoff, 45,46 one can use the pseudospectrum to obtain the photoionization cross section via the so-called Stieltjes imaging approach. ${ }^{67}$ One considers the moments $s(k)$ of the oscillator strength, defined

$$
s(k)=\sum_{j}^{\text {discr }} \omega_{j}^{k} f_{j}+\int_{\omega_{T}}^{\infty} \omega^{k} f(\omega) d \omega
$$

which are central quantities in the theory of moments. It is assumed that the moments of the continuum oscillator strength, $S(k)=\int_{\omega_{T}}^{\infty} \omega^{k} f(\omega) d \omega$, can be approximated as a sum of $N$ discrete pseudostates that fall into the continuum, $S(k) \approx \sum_{i}^{N} \bar{\omega}_{i}^{k} \bar{f}_{i}$.

In Stieltjes imaging, a "primitive" set of $M$ excitation energies and oscillator strengths is transformed in order to obtain the correct representation of the continuum and the energy normalization. One first computes a number $(2 r)$ of "spectral moments" $S(-k)$ of the primitive spectrum

$$
S(-k)=\sum_{j=1}^{M} f_{j} \omega_{j}^{-k}, \quad k=0, \ldots, 2 r-1,
$$

where $r$ is chosen a priori. ${ }^{56}$ As the pseudostates of the primitive spectrum come from a calculation in the $L^{2}$ basis, they have, above the ionization threshold, the wrong boundary conditions, so they are physically meaningless and extremely method and basis-set dependent. However, as the basis set approaches completeness, their spectral moments $S(-k)$ converge toward the spectral moments of the true oscillator strength.

From the $2 r$ spectral moments, discretized spectra of order $n=2, \ldots, r$, called principal pseudospectra, are generated as generalized (Gaussian) quadrature points (abscissae) $\omega_{j}^{(n)}$ and weights $f_{j}^{(n)}$,

$$
S(-k)=\sum_{j=1}^{n} f_{j}^{(n)}\left(\omega_{j}^{(n)}\right)^{-k}, \quad k=0, \ldots, 2 n-1 .
$$

Proceeding in this way, the cross section is "smoothed," i.e., there are typically many more "raw" pairs of energies and oscillator strengths in the primitive spectrum than quadrature points and weights in the principal spectrum. These resulting points are adapted to reproduce the lowest order spectral moments, which should be the ones more accurately reproduced by the primitive set. The Stieltjes process also places the points where they are most needed, that is, 
the density of abscissae is higher where the cross section changes more rapidly.

From the Gaussian quadrature points $\omega_{i}$ and weights, $f_{i}$ one constructs an approximate distribution function $F^{(n)}$ of order $n$,

$$
\begin{aligned}
& F^{(n)}(\omega)=0, \quad \omega<\omega_{1}, \\
& F^{(n)}(\omega)=\sum_{j=1}^{i} f_{j}, \quad \omega_{i}<\omega<\omega_{i+1}, \\
& F^{(n)}(\omega)=\sum_{j=1}^{n} f_{j}=S(0), \quad \omega_{n}<\omega .
\end{aligned}
$$

$F^{(n)}(\omega)$ is equivalent to a cumulative oscillator strength for the continuum range. Its differentiation yields an $n$th order approximate density function $\tilde{f}(\omega)$, known as Stieltjes density. This differentiation can be done in many ways. According to one of the most common recipes, the continuum oscillator strength density is computed pointwise (i.e., numerically) as the Stieltjes derivative

$$
\tilde{f}(\omega)=\frac{d F^{(n)}}{d \omega^{(n)}}=\frac{1}{2} \frac{f_{i+1}^{(n)}+f_{i}^{(n)}}{\omega_{i+1}^{(n)}-\omega_{i}^{(n)}}
$$

at energy

$$
\omega=\frac{1}{2}\left(\omega_{i+1}^{(n)}+\omega_{i}^{(n)}\right) .
$$

Finally, the ionization cross section is calculated from Eq. (9) employing $\tilde{f}(\omega)$ so that each order $n$ gives $n-1$ points on the cross section curve. These can be interpolated to yield a continuous curve.

\section{The XAS cross section below and above the ionization limit from the analytic continuation procedure based on the Padé approximants}

Photoabsorption cross sections, $\sigma(\omega)$, can be obtained from the imaginary part of the averaged dynamic dipole polarizability function, $\bar{\alpha}(\omega)$,

$$
\sigma(\omega)=\frac{4 \pi \omega}{c} \lim _{\eta \rightarrow 0} \operatorname{Im}[\bar{\alpha}(\omega+i \eta)] .
$$

We demonstrated in previous works ${ }^{58,59,61}$ that an analytic continuation procedure could be applied to either TDDFT or CC electronic pseudospectra in the VUV and inner-shell frequency regions to build an analytic complex valued expression for the averaged dynamic dipole polarizability. The real and imaginary parts of the dynamic dipole polarizability expression can be easily separated and the photoabsorption cross section directly obtained from its imaginary part. finite sum

Briefly, our methodology consists in approximating ${ }^{68} \alpha(z)$ by a

$$
\alpha(z)=\sum_{i=1}^{k} \frac{f_{i}}{\omega_{i}^{2}+z^{2}},
$$

where the electronic spectrum $\left\{\omega_{i}, f_{i}\right\}_{i=1, k}$ stands for the transition frequencies and oscillator strengths obtained in a $L^{2}$ basis set calculation and $z$ is the complex valued frequency.
Having the electronic pseudospectrum at hand, we construct the approximation for $\alpha(z)$ as in Eq. (22) and calculate $\alpha(z)$ at a number of points in the complex plane. These points are fitted by a continued fraction-which its mathematical structure is equivalent to the Padé approximants, providing a representation of $\alpha(z)$ in the complex plane. Using this representation, from a converged continued fraction, we calculate $\alpha(z)$ on the real axis where it equals $\alpha(\omega)$. The imaginary part of $\alpha(z)$ on the real axis provides the cross section by Eq. (21). Details can be found elsewhere ${ }^{58,61}$ and references therein.

The set of complex frequency points at which the function in Eq. (22) is interpolated is arbitrary and should span the whole frequency domain of the calculated spectrum. To construct the approximation in Eq. (22), the set of transition energies and oscillator strengths have been used, i.e., $\left\{\omega_{i}, f_{i}\right\}_{i=1, \Lambda}$, where $\Lambda$ stands for the length of the CVS-CC pseudospectrum.

In the spirit of Ref. 59, the set of complex frequencies $\left\{z_{j}\right\}$ has been chosen as follows: the real part $x_{j}$ is made equal to the half value of two successive transition frequencies $\left(\omega_{j}+\omega_{j+1}\right) / 2$ and the normalized cumulative oscillator strength $F_{j}=\sum_{r=1}^{j} \frac{f_{r}}{S(0)}$ is taken as the imaginary part $y_{j}$.

\section{Computational details}

The experimental equilibrium structures of the systems considered, namely, ethylene, carbon dioxide, water, ammonia, acetaldehyde, furan, and pyrrole, obtained from the NIST web page ${ }^{69}$ were used. The CVS scheme of Ref. 42, applied to the asymmetric Lanczos procedure for the calculation of the spectral moments within the CCLR ${ }^{41}$ as implemented in the Dalton program package, ${ }^{70}$ was used to obtain the CC2 and CCSD core-level pseudospectra. Chain-length subspaces of $J=250$ and 500 were used. The pseudospectra were subsequently fed into the Stieltjes imaging procedure ${ }^{56,57}$ and the analytic continuation procedure based on Padé approximants functions, ${ }^{61}$ to generate the continuum part of the cross section profiles. Both the Stieltjes imaging and the analytic continuation procedures were implemented as stand-alone Fortran 90 codes. ${ }^{56,61}$

Stieltjes imaging is known to be susceptible to numerical errors due to the fact that it involves recursive calculation of a number of quantities. As the positive spectral moments $S(k)$ diverge for $k>2$, Stieltjes imaging was performed using the negative spectral moments. Stieltjes orders of 2-20 were typically computed. ${ }^{56,57}$ However, only those which are well converged (indicated in the figures) were interpolated to obtain the X-ray photoionization cross sections. Cubic spline interpolation was used in such cases.

Depending on the case at hand, Dunning's correlation consistent basis sets aug-cc-pVTZ and aug-cc-pVDZ ${ }^{71}$ and Pople's ${ }^{72}$ $6-311++G^{* *}$ basis set were employed. The proper description of the continuum region is a key goal of the present study since it is extremely sensitive to the $L^{2}$ basis set. An adequate description of this region has been shown to be attainable ${ }^{56-58}$ by using the continuumlike basis functions proposed by Kaufmann, Baumeister, and Jungen. ${ }^{73}$ Therefore, sets of $s, \mathrm{p}, \mathrm{d}$, and $\mathrm{f}$ continuumlike functions, generated according to the prescriptions of Kaufmann, Baumeister, and Jungen, ${ }^{73}$ were added to the center of mass of each molecule considered. We have tested different continuum sets by varying the 
quantum numbers $n$ in the Kaufmann recipe and found the set with $n=2, \ldots, 8$, a good compromise between accuracy and computational cost. Therefore, we will report and discuss here the results obtained using Kaufmann's quantum numbers $n$ ranging from 2 up to 8 . A notationlike $(7 \mathrm{~s} 7 \mathrm{p} 7 \mathrm{~d} . . .)_{n=2-8}$ will be used throughout the paper to indicate the selected set of Rydberg functions for each molecular system.

In Figs. 1-14, the purple line refers to the cross section obtained via the analytic continuation procedure based on Padé approximants, which treats the discrete and the continuum parts of the spectrum on the same footing and therefore covers both the regions below and above the ionization energy. The orange line refers to the cross section generated via Stieltjes imaging, which only applies to the continuum part of the spectrum. A Lorentzian broadening of the discrete stick spectrum is also used to simulate the cross section below the ionization limit and is represented by the light blue line. The experimental ionization energies (IEs) as well as the calculated ones will be shown as vertical dashed lines and their values are given in the corresponding figure captions. The computed cross sections and IEs have been completely rigidly shifted by the value $(\Delta)$ indicated in the figure captions, in order to match the first computed peak of the XAS spectrum with the maximum of the first peak in the corresponding experiment.

The experimental data points were either digitized from the original reference using WebPlotDigitizer ${ }^{74}$ or recovered directly from Hitchcock and Mancini's database. ${ }^{75,76}$ The spectral signals reported on the database refer only to the contribution from the core excitations, i.e., the underlying signals from ionization of valence electrons have been removed by extrapolating the pre-edge signal, which is in essence what the CVS scheme also attains.

\section{RESULTS}

\section{A. Ethylene}

In Figs. 1 and 2, we present the inner-shell cross sections at the carbon $\mathrm{K}$-edge of $\mathrm{C}_{2} \mathrm{H}_{4}$, along with the experimental one taken from Ref. 77 (see also Ref. 75).

Figures 1(a) and 1(b) show the CVS-CCSD results obtained within the aug-cc-pVTZ+(7s7p7d $)_{n=2-8}$ basis set, for two different values of the chain length, namely, $J=250$ and $J=500$. Figure 1 (c) shows the CVS-CC2 results obtained in the same basis set and for $J=250$, whereas in Fig. 1(d), we report the CVS-CCSD cross sections obtained with a slightly larger Rydberg set including $f$ functions. In Figs. 2(a) and 2(b), the cross sections obtained with the $6-311++\mathrm{G}^{* *}$ basis set and two different Rydberg sets are also shown.

The experimental cross section ${ }^{77}$ of ethylene presents three main peaks below the ionization threshold assigned as follows: the first one, at $284.7 \mathrm{eV},{ }^{77}$ to a $1 \mathrm{~s}_{\mathrm{C}} \rightarrow \pi^{*}$ transition, the second one, at $287.8 \mathrm{eV}$, to a $1 \mathrm{~s}_{\mathrm{C}} \rightarrow 3 s, 3 p$ transitions, and the third one, at $289.3 \mathrm{eV}$, to a $1 \mathrm{~s}_{\mathrm{C}} \rightarrow 4 p$ transition. As it can be observed in Figs. 1(a) and 1(b), the cross section below the IE limit as well as the continuum tail is already converged at $J=250$, as no significant differences can be seen in the $J=250$ and $J=500$ spectra. This is an indication that the chain length $J=250$ is sufficiently large to treat the discrete as well as the continuum regions of the spectra. Both Padé and Stieltjes procedures yield cross section profiles well above the ionization limit of comparable quality and are in reasonable good agreement with the experimental data. As already observed in a recent study by some of us, ${ }^{61}$ in the region below the IE, the Padé procedure is unable to describe all of the experimental spectral features, which, on the other hand, are neatly obtained by the Lorentzian broadening of the Lanczos sticks. In the Padé analytic continuation procedure, nonetheless, the discrete and the continuum cross sections are continuously connected, due to the analytic treatment, avoiding the discontinuity at the threshold connecting the discrete spectrum obtained by Lorentzian broadening and the continuum part provided by Stieltjes imaging. Inclusion of additional continuumlike functions of $\mathrm{f}$ character has a moderate, but still noticeable, effect especially on the Padé results, slightly improving the continuum tail above $305 \mathrm{eV}$, but enhancing the broad band at around $298 \mathrm{eV}$ [see Fig. 1(d)].

As for the comparison between CVS-CCSD and CVS-CC2, it is evident from Figs. 1(a) and 1(c) that, for the K-edge of ethylene, CVS-CC2 is less accurate than CCSD both below and above the IE limit, as well as the IE value itself, most probably due to the lack of description of double excitations, which are important to account for orbital relaxation.

The Pople set $6-311++\mathrm{G}^{* *}$ is a rather popular choice of basis set for XAS calculations, ${ }^{35,43,78}$ and in Fig. 2(a), we show how it performs when coupled with the same continuumlike ${ }^{73}$ sets and $J$ value used in Figs. 1(a) and 1(d). Up to the IE, the main differences observed when compared to the aug-cc-pVTZ results are in the positions of the $1 \mathrm{~s}_{\mathrm{C}} \rightarrow \pi^{*}$ peak and of the IE. The energy difference between the experimental and the calculated CVS-CCSD $1 \mathrm{~s}_{\mathrm{C}} \rightarrow \pi^{*}$ transition is $1.27 \mathrm{eV}$ for the $6-311++\mathrm{G}^{* *}$ basis set, vs $0.83 \mathrm{eV}$ for the augcc-pVTZ basis. The IE is found at $292.0 \mathrm{eV}$, vs $291.6 \mathrm{eV}$ of the aug-cc-pVTZ basis. The $6-311++\mathrm{G}^{* *}$ set is thus slightly less accurate than the aug-cc-pVTZ basis set up to the IE limit. Above the IE, one observes more clustered groups of discrete pseudoexcited states than those obtained with the Dunning set, which can possibly explain the presence of slight oscillations in the cross section from the analytic continuation procedure. After increasing the continuumlike set with extra functions, i.e., $(7 \mathrm{~s} 7 \mathrm{p} 7 \mathrm{~d} 7 \mathrm{f})_{n=2-8}$, the calculated cross section in Fig. 2(b) is similar to the one of Fig. 1(d), with an improved continuum tail above $305 \mathrm{eV}$ when compared to experiment, but with the appearance of a broad band at around $298 \mathrm{eV}$ that is not present in the experimental cross section. The $6-311++\mathrm{G}^{* *}$ basis set should then probably be used with some care when aiming at the photoionization part of the XAS cross section.

\section{B. Carbon dioxide}

The photoabsorption/photoionization cross sections of $\mathrm{CO}_{2}$ at the $\mathrm{O}$ and $\mathrm{C}$ K-edges are shown in Figs. 3 and 4, respectively. In Figs. 3(a) and 3(b), we show the cross sections obtained at the CVS-CCSD and CVS-CC2 levels, respectively, via the analytic continuation procedure (purple line) as well as by Lanczos broadening (blue line) plus Stieltjes imaging (orange line). In all calculations, the aug-cc-pVTZ basis set supplemented with the $(7 \mathrm{~s} 7 \mathrm{p} 7 \mathrm{~d})_{n=2-8}$ continuumlike set $^{73}$ was adopted, along with a Lanczos chain length $J=500$. The computed cross sections are compared to the experimental results of electron energy loss spectroscopy (EELS) under the electric dipole scattering condition, obtained by McLaren et al. 


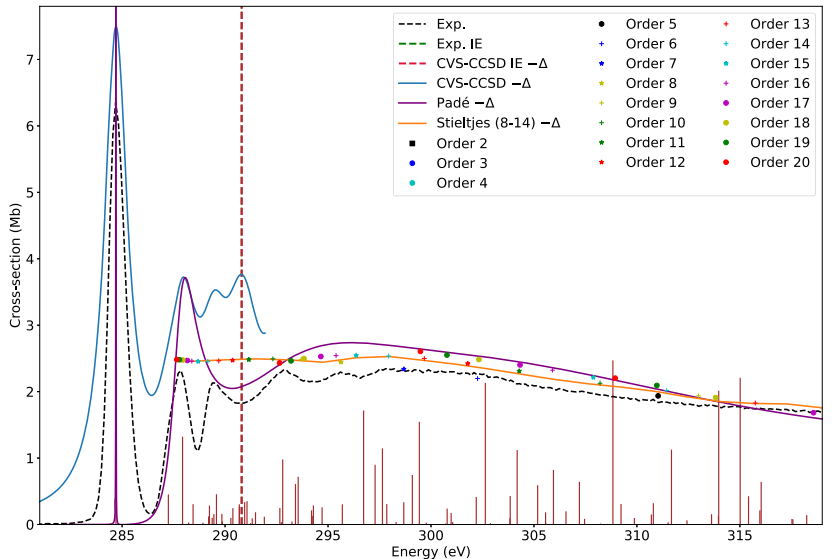

(a)

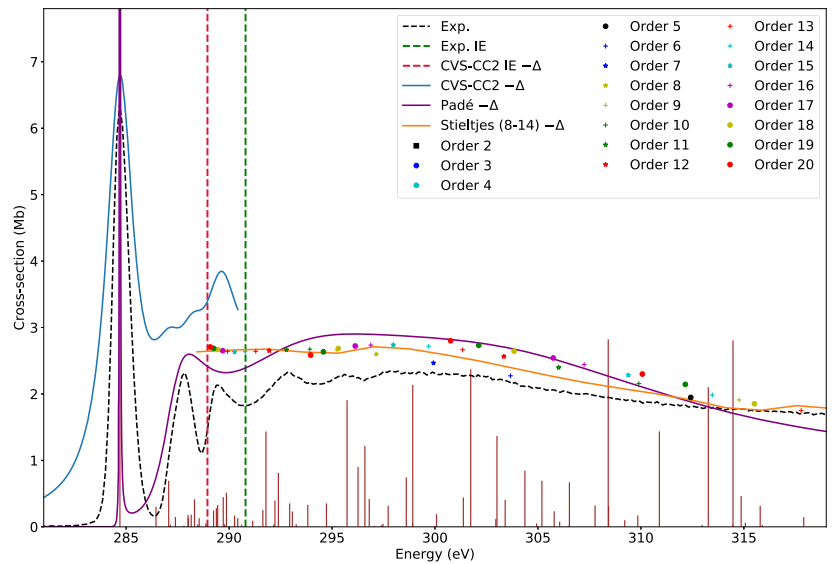

(c)

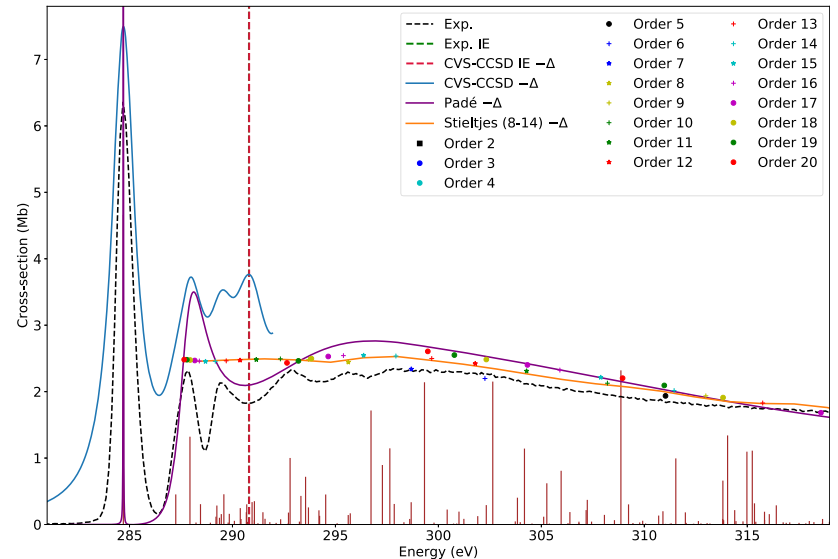

(b)

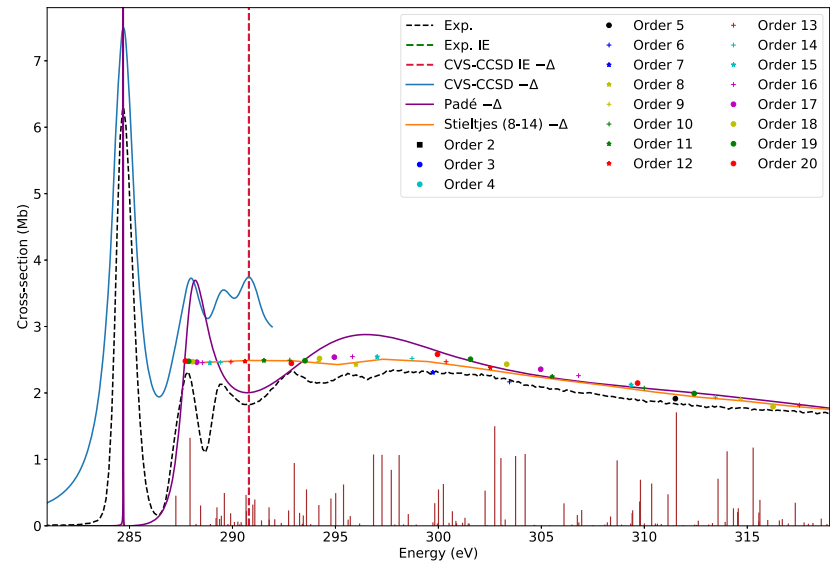

(d)

FIG. 1. Ethylene. Carbon K-edge photoabsorption cross sections obtained with two different choices of basis set and chain length $\mathrm{J}$. The discrete peaks were broadened with a Lorentzian function, HWHM $=0.68 \mathrm{eV}$ for CVS-CCSD and HWHM $=0.81$ for CVS-CC2. The calculated spectra were shifted by $\Delta=0.83 \mathrm{eV}$ in panels (a) and (b), by $\Delta=2.34 \mathrm{eV}$ in panel (c), and by $\Delta=0.84 \mathrm{eV}$ in panel (d). Experimental results are from Refs. 75 and 77 . (a) CVS-CCSD aug-cc-pVTZ $+(7 \mathrm{~s} 7 \mathrm{p} 7 \mathrm{~d})_{n=2-8}, J=250 ;$ (b) CVS-CCSD aug-cc-pVTZ+(7s7p7d) $n_{n=2-8}, J=500 ;$ (c) CVS-CC2/aug-cc-pVTZ+(7s7p7d $)_{n=2-8}, J=250$; and (d) CVS-CCSD/aug-cc-pVTZ+(7s7p7d7f $)_{n=2-8}, J=500$.

Below the $1 \mathrm{~s}_{\mathrm{O}}^{-1}$ ionization threshold, the $\mathrm{CO}_{2}$ cross section presents an intense peak centered at $535.4 \mathrm{eV}$ due to a $1 \mathrm{so} \rightarrow \pi^{*}$ transition. The energy difference $\Delta$ between the experimental and the calculated $1 \mathrm{so} \rightarrow \pi^{*}$ transition is $1.56 \mathrm{eV}$ and $0.05 \mathrm{eV}$ at the CVS-CCSD and CVS-CC2 levels, respectively. The IEs are found at $542.9 \mathrm{eV}$ for CVS-CCSD and $539.0 \mathrm{eV}$ for CVS-CC2, vs the experimental value of $541.3 \mathrm{eV}$. Even if the systematic shift $\Delta$ is smaller in the CVS-CC2 case than for CVS-CCSD, the agreement between the shifted CVS-CCSD ionization threshold and the experimental value (see vertical dashed lines in Fig. 3) is better than in the CVS-CC2 case.

Above the ionization threshold, the experimental continuum cross section exhibits a broad band with an offset at around $560 \mathrm{eV}$, with a maximum of $1.7 \mathrm{Mb}$, after which it decreases for a nearly constant plateau of roughly $1 \mathrm{Mb}$.

The broad feature above $560 \mathrm{eV}$ and the continuum tail up to $620 \mathrm{eV}$ are well described by both procedures, whose overall spectral features are very similar to each other. Comparing Figs. 3(a) and $3(\mathrm{~b})$, we note that CVS-CCSD and CVS-CC2 yield rather similar (shifted) cross sections for the O K-edge of $\mathrm{CO}_{2}$, with CVS-CCSD marginally more accurate than the CVS-CC2 one.

The $\mathrm{C}$ K-shell cross sections of $\mathrm{CO}_{2}$ are presented in Fig. 4 along with experimental results. ${ }^{77}$ The strong absorption peak at $290.8 \mathrm{eV}$ is known to originate from the $1 \mathrm{~s}_{\mathrm{C}} \rightarrow \pi^{*}$ transition, ${ }^{77,}$ which we found at $0.33 \mathrm{eV}$ and $1.68 \mathrm{eV}$ higher in energy at CVSCCSD and CVS-CC2 levels, respectively. Opposite to the O K-edge case, the shift $\Delta$ is thus smaller for CCSD than for CC2. Also different from the O K-edge case, the shifted CC2 IE is closer to the experimental value than the CCSD one.

The calculated cross sections show, similar to the oxygen edge, that the Stieltjes and the Padé approximants procedures yield very similar photoionization cross sections above the IE limit. While the computed cross sections follow rather closely the experimental one up to $360 \mathrm{eV}$, at higher energies, and, in particular, near $380 \mathrm{eV}$, a 


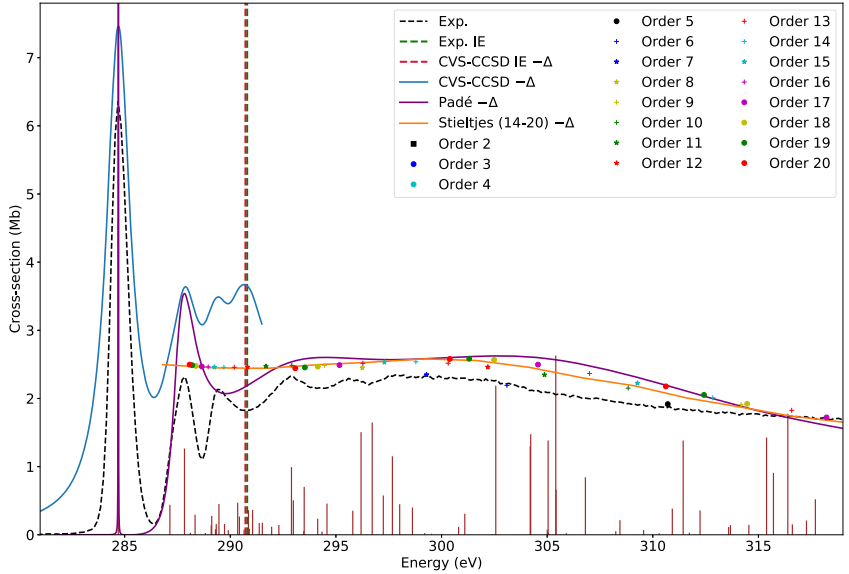

(a)

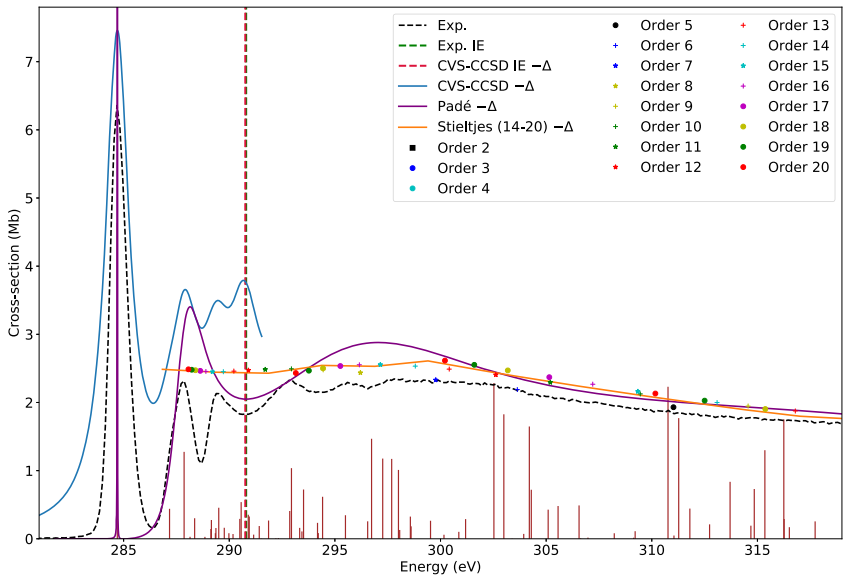

(b)

FIG. 2. Ethylene. CVS-CCSD carbon K-edge photoionization cross sections obtained with $J=250$. The $6-311++G^{* *}$ basis set was supplemented with the continuumlike sets $(7 \mathrm{~s} 7 \mathrm{p} 7 \mathrm{~d})_{n=2-8}$ [panel (a)] and $(7 \mathrm{~s} 7 \mathrm{p} 7 \mathrm{~d} 7 \mathrm{f})_{n=2-8}$ [panel (b)]. The discrete peaks were broadened with a Lorentzian function, HWHM $=0.68 \mathrm{eV}$. The calculated spectra were shifted by $\Delta=1.27 \mathrm{eV}$ in panels (a) and (b). Experimental results are from Refs. 75 and 77 . (a) $6-311++G^{* *}+(7 \mathrm{~s} 7 \mathrm{p} 7 \mathrm{~d})_{n=2-8}, J=250$ and $(\mathrm{b}) 6-311++\mathrm{G}^{* *}+(7 \mathrm{~s} 7 \mathrm{p} 7 \mathrm{~d} 7 \mathrm{f})_{n=2-8}$, $J=250$.

broad feature arises in the computed profiles, which is not observed in the experiment.

\section{Water}

The calculated CVS-CCSD and CVS-CC2 oxygen K-shell photoionization cross sections of water are shown in Fig. 5 along with the experimental EELS results obtained by Ishii et al. ${ }^{75,80}$ The augcc-pVTZ basis supplemented with the Kaufmann ${ }^{73}(7 \mathrm{~s} 7 \mathrm{p} 7 \mathrm{~d})_{n=2,8}$ continuumlike set was used, and the Lanczos chain was truncated at
$J=500$. As in the previous cases, the continuum part of the spectra shown in Figs. 5(a) and 5(b) was treated with both the Padé analytic continuation procedure and Stieltjes Imaging, while the discrete sticks were broadened with Lorentzian functions with HWHM $=0.54 \mathrm{eV}$ for CCSD and $0.41 \mathrm{eV}$ for CC2.

The experimental peaks below the oxygen K-edge ionization threshold have been extensively studied at different levels of theory, such as CCSD, ${ }^{40,43,61}$ TDDFT $^{61}$ and $\operatorname{ADC}(2) .{ }^{34}$ The first two bands centered at 534.0 and $535.9 \mathrm{eV}$ have been assigned to the $1 \mathrm{~s}_{\mathrm{O}} \rightarrow 3 \mathrm{~s}$ and $1 \mathrm{~s}_{\mathrm{O}} \rightarrow 3 \mathrm{p}$ transitions. The width of these bands

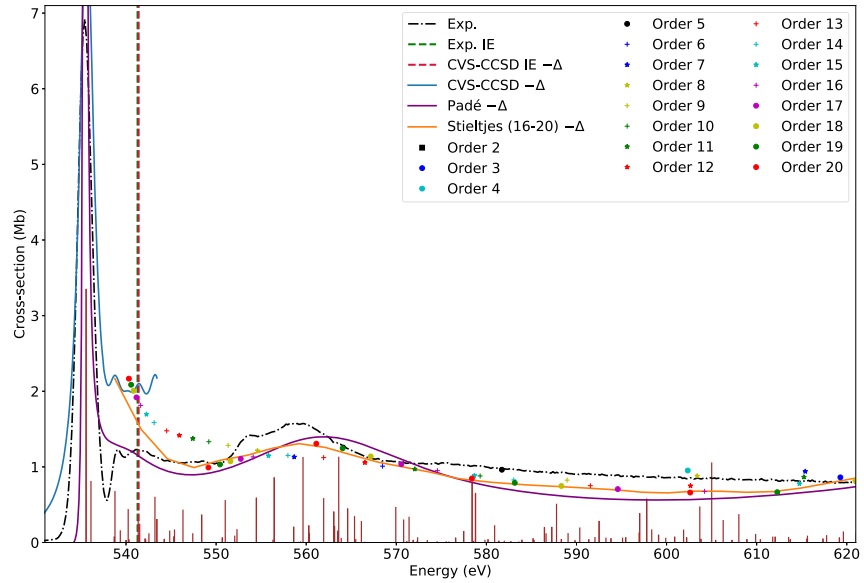

(a)

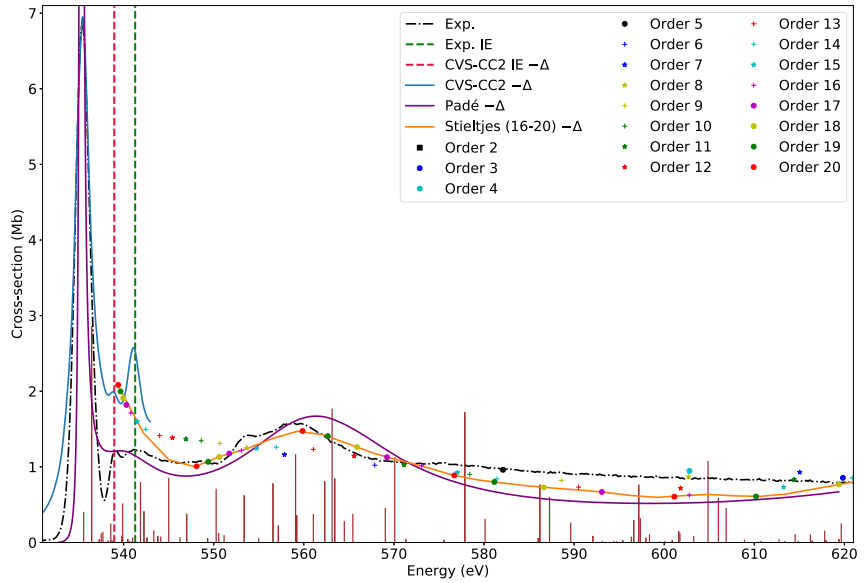

(b)

FIG. 3. $\mathrm{CO}_{2}$. CVS-CCSD (a) and CVS-CC2 (b) oxygen K-edge cross sections obtained with $J=500$ in the aug-cc-pVTZ+(7s7p7d $)_{n=2-8}$ basis set. The discrete peaks were broadened with a Lorentzian function, HWHM $=0.95 \mathrm{eV}$. The calculated spectra were shifted by $\Delta=1.56 \mathrm{eV}$ [panel (a)] and $\Delta=0.05 \mathrm{eV}$ [panel (b)]. Experimental results taken from Refs. 75 and 77. 


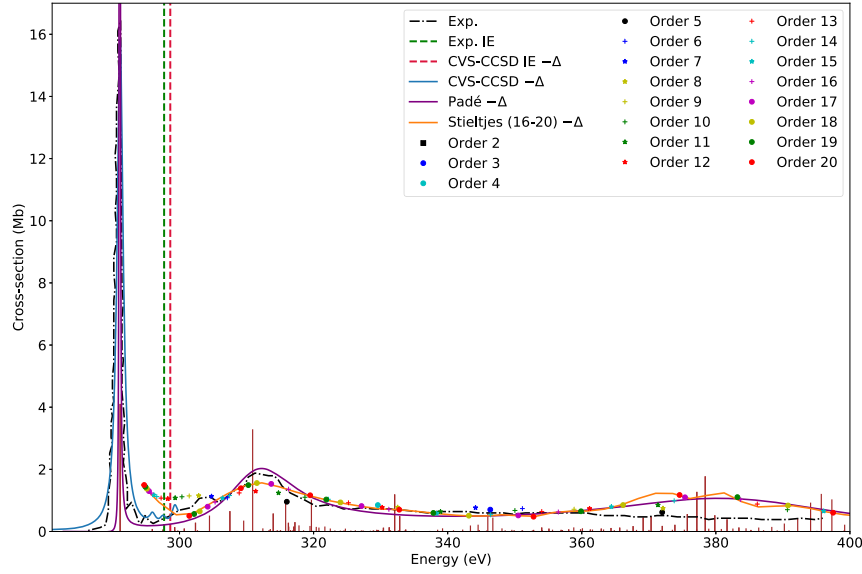

(a)

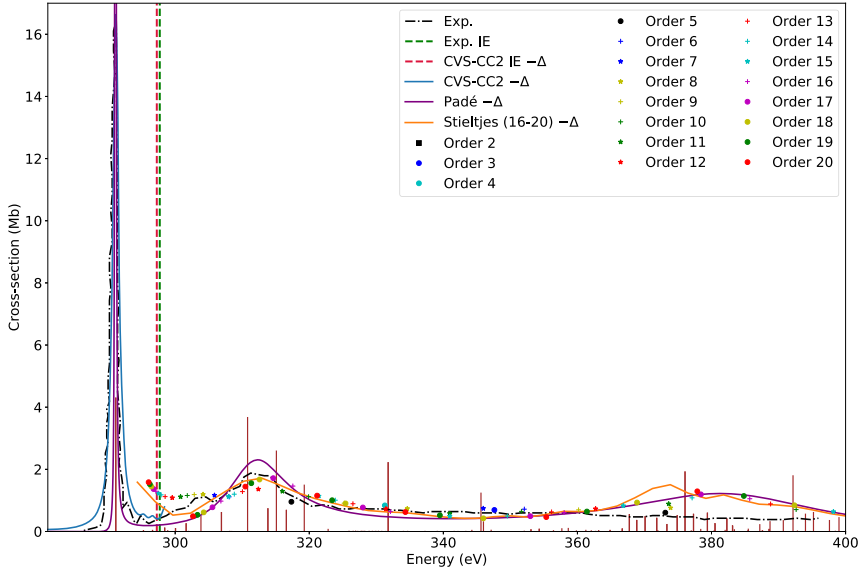

(b)

FIG. 4. $\mathrm{CO}_{2}$. CVS-CCSD (a) and CVS-CC2 (b) carbon K-edge photoionization cross sections obtained with $\mathrm{J}=500$ and the aug-cc-pVTZ basis set, supplemented with a $(7 \mathrm{~s} 7 \mathrm{p} 7 \mathrm{~d})_{n=2-8}$ continuumlike set. ${ }^{73}$ The discrete peaks were broadened with a Lorentzian function, HWHM $=0.54 \mathrm{eV}$. The calculated spectra were shifted by $\Delta=0.33 \mathrm{eV}$ [panel (a)] and $\Delta=1.68 \mathrm{eV}$ [panel (b)]. Experimental results taken from Refs. 75 and 77.

indicates that both excitations have dissociative character. The third band centered at $537.0 \mathrm{eV}$ has been attributed to the $1 \mathrm{~s}_{\mathrm{O}} \rightarrow 3 \mathrm{p}\left(a_{1} / b_{1}\right)$ transition, and the remaining weaker bands located at 537.9 and $538.5 \mathrm{eV}$ were assigned to members of the $1 \mathrm{~s}_{\mathrm{O}} \rightarrow \mathrm{ns}$ and $1 \mathrm{~s}_{\mathrm{O}} \rightarrow \mathrm{np}$ Rydberg series. The first three features are also described by the present CVS-CCSD and CVS-CC2 calculations, though with quite different relative intensities, as also observed previously.

The shifted CVS-CCSD ionization threshold (dashed red line) is clearly in better agreement with the experimental value at $539.7 \mathrm{eV}$ (see the caption in Fig. 5) than the (shifted) CVS-CC2 ionization energy.
Above the IE, the photoionization cross sections yielded by both continuum procedures and CC approximations noticeably deviate from the experimental result in the region between 540 and $555 \mathrm{eV}$. The Padé cross section, in particular, shows a slight oscillatory trend, which is smoothed in the Stieltjes case. As also shown in a previous study, ${ }^{61}$ the cross section below the IE threshold obtained with the Padé approximants only accounts for the two intense peaks dominated by the $1 \mathrm{~s}_{\mathrm{O}} \rightarrow 3 \mathrm{~s}$ and $1 \mathrm{~s}_{\mathrm{O}} \rightarrow 3 \mathrm{p}$ transitions.

The present CVS-CC photoionization cross sections obtained with both Stieltjes and Padé procedures within the CVS-Lanczos framework are similar to the full-space results reported in Ref. 61.

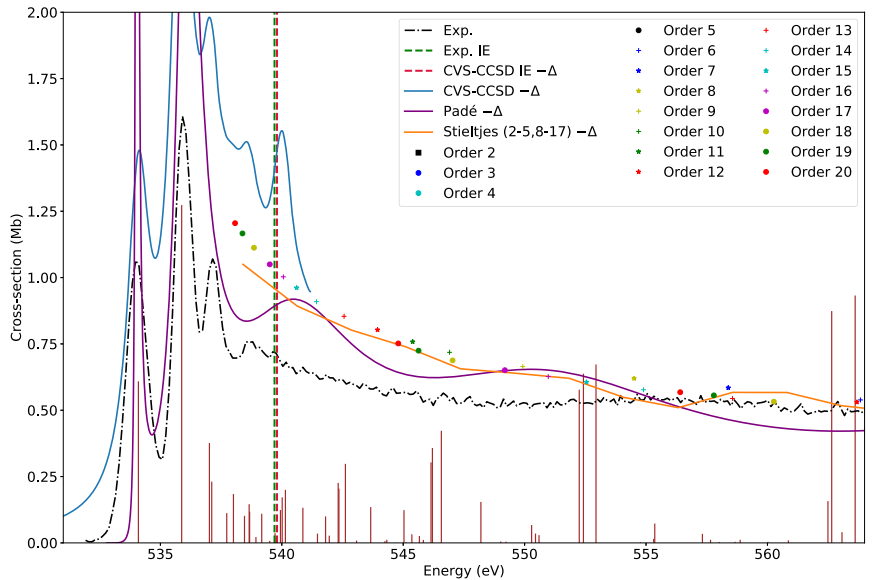

(a)

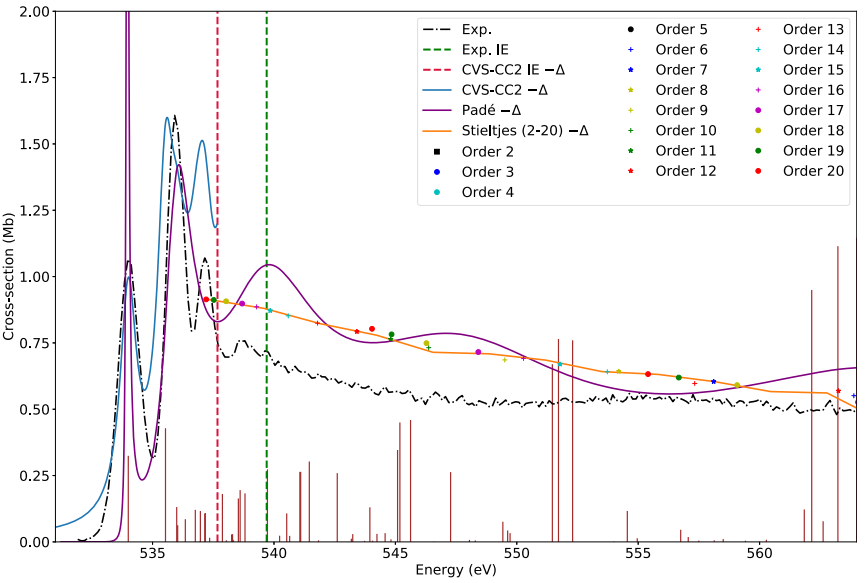

(b)

FIG. 5. Water. CVS-CCSD (a) and CVS-CC2 (b) oxygen K-edge cross sections obtained with $J=500$ and the aug-cc-pVTZ+(7s7p7d) $n=2,8$ basis set. The discrete peaks were broadened with a Lorentzian function, HWHM $=0.54 \mathrm{eV}$ [panel (a)] and $0.41 \mathrm{eV}$ [panel (b)]. The calculated spectra are shifted by $\Delta=1.20 \mathrm{eV}[$ panel (a)] and $\Delta=0.06 \mathrm{eV}$ [panel (b)]. Experimental results are from Ref. 80. 


\section{Ammonia}

Experimental K-edge spectra of ammonia were obtained by Sodhi and Brion ${ }^{81}$ with electron energy loss spectroscopy under the electric dipole scattering condition and by Schirmer et al. ${ }^{34}$ with synchrotron radiation, both reported in arbitrary units. In Fig. 6, the photoabsorption cross sections of ammonia obtained at the CVSCCSD and CVS-CC2 levels of calculation are presented together with the experimental EELS data by Sodhi and Brion. ${ }^{81}$ The calculations were performed in the aug-cc-pVTZ basis $+(7 \mathrm{~s} 7 \mathrm{p} 7 \mathrm{~d})_{n=2-8}$ continuumlike set. The Lanczos chain was truncated at $J=500$. Since the experimental cross sections were given in arbitrary scale, the experimental photoionization tail was adjusted to match the theoretical curve. The photoabsorption cross section of ammonia presents four distinct peaks below the IE: the first one, centered at $400.6 \mathrm{eV}$, has been attributed to the $1 \mathrm{~s}_{\mathrm{N}} \rightarrow 3 \mathrm{~s}$ transition, the second one at $402.3 \mathrm{eV}$ has been assigned to the $1 \mathrm{~s}_{\mathrm{N}} \rightarrow 3 \mathrm{p}(e)$ transition, the third one centered at $402.8 \mathrm{eV}$ to the $1 \mathrm{~s}_{\mathrm{N}} \rightarrow 3 \mathrm{p}\left(a_{1}\right)$ transition, and the fourth one, at $403.6 \mathrm{eV}$, to the $1 \mathrm{~s}_{\mathrm{N}} \rightarrow 4 \mathrm{~s}$ transition. ${ }^{34,40}$ The discrete part of the spectrum presented in Fig. 6(a), obtained by Lorentzian broadening the discrete CCSD sticks with $\mathrm{HWHM}=0.27 \mathrm{eV}$, describes the above-mentioned experimental features, in agreement with previous studies. ${ }^{41,61}$ As also discussed in Ref. 61, the analytic continuation procedure applied to the discrete region does not have enough resolution to distinguish all the discrete peaks and, normally, only the most intense transitions, in this case the $1 \mathrm{~s}_{\mathrm{N}} \rightarrow 3 \mathrm{~s}$ and the $1 \mathrm{~s}_{\mathrm{N}} \rightarrow 3 \mathrm{p}$ transitions, are obtained.

The shifted $1 \mathrm{~s}_{\mathrm{N}}^{-1}$ IE CVS-CCSD ionization energy (dashed red line) is in very good agreement with the experimental value $(405.6 \mathrm{eV})$, while the shifted CC2 one IE is significantly underestimated, as also observed for the $\mathrm{O}$ and $\mathrm{C}$ IEs in the previously discussed systems.

Above the IE, our results show that the continuum parts of the spectra treated with both continuum procedures and CC levels are in very good agreement with each other and with the experimental result but one should keep in mind that the experiment has been scaled to match the computed continuum tail.

As previously mentioned, a recent study by some of us ${ }^{61}$ reported a theoretical description of the $1 s_{\mathrm{N}}$ cross section spectrum of the ammonia molecule calculated at the TDDFT as well as the CCS, CC2, and CCSD levels of theory in full space. Reference 61 indicated that the CCSD photoionization cross sections are more accurate compared with the results obtained at the other levels of theory there employed.

\section{E. Full space vs CVS photoionization cross sections: The ammonia and ethylene cases}

In Fig. 7, we compare the photoionization cross sections of ammonia and ethylene obtained via a full-space CCSD calculation (blue lines) with the CVS-CCSD cross sections (purple lines) that were also shown in Figs. 6(a) and 1(a). In the full-space calculation, all types of occupied orbitals are allowed to participate in the manifold of excitations out of the reference state. Similarly to what was done in Ref. 61 for ammonia, the Lanczos chain length was truncated at $J=3000$ and the same basis set of Figs. 6(a) and 1(a) was used. For both molecules, the full space calculation roughly reproduces the same features obtained in the CVS calculation. However, in the case of ammonia, one can notice the appearance of a broad band centered at $409 \mathrm{eV}$ and a more oscillating continuum tail, compared with the CVS result. The oscillatory behavior of the fullspace photoionization cross section is most probably related to the inclusion of the excitations of valence electrons in the X-ray region, giving rise to a "background noise" of pure valence excitations into the core-excitation region. This background noise is specifically removed via the CVS scheme. This behavior indicates that the Padé procedure is very sensitive to small changes in the pseudospectrum. We also note in passing that Stieltjes imaging cannot be straightforwardly applied to the full-space stick spectrum unless

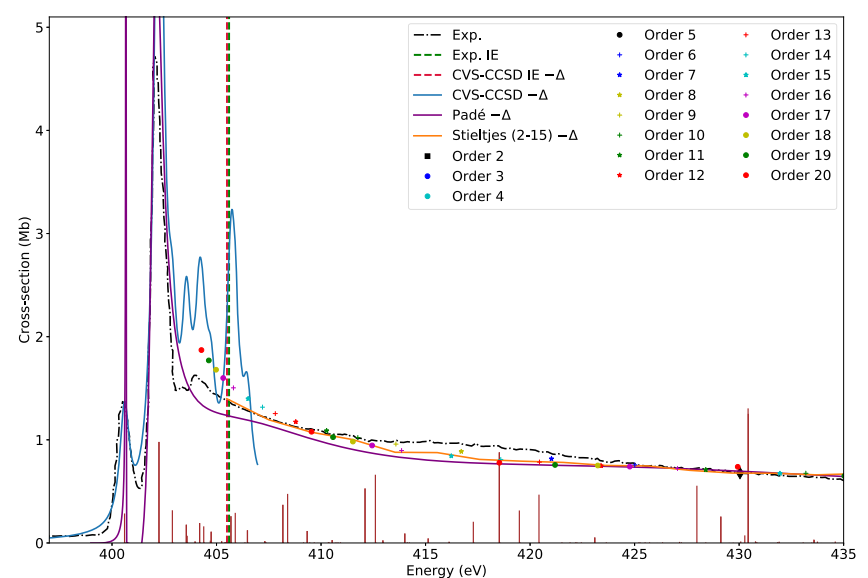

(a)

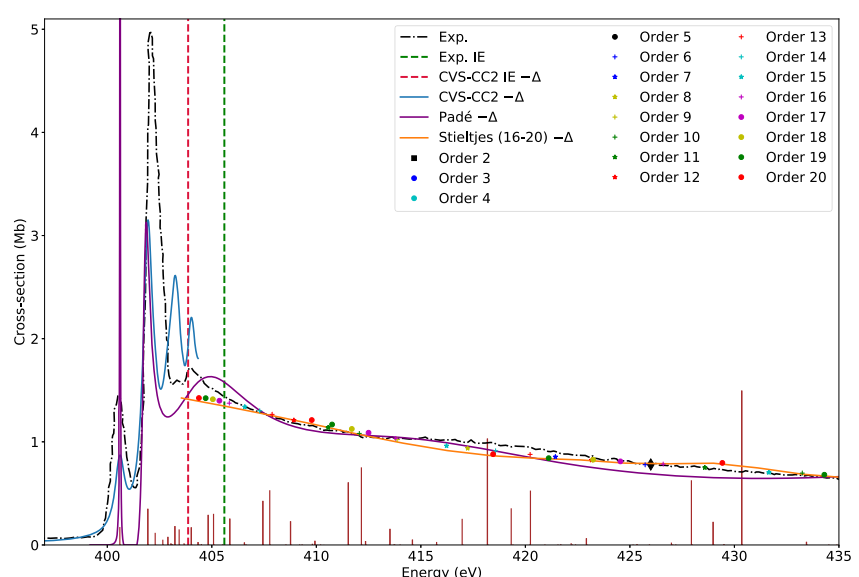

(b)

FIG. 6. $\mathrm{NH}_{3}$. CVS-CCSD [panel (a)] and CVS-CC2 [panel (b)] nitrogen K-shell cross sections obtained with $J=500$ and the aug-cc-pVTZ+(7s7p7d $)_{n=2-8}$ basis set. The discrete peaks were broadened with a Lorentzian function, HWHM $=0.27 \mathrm{eV}$. The calculated CVS-CCSD spectra were shifted by $\Delta=1.02 \mathrm{eV}$ [panel (a)] and the CVS-CC2 ones by $\Delta=0.83 \mathrm{eV}$ [panel (b)]. Experimental results are from Ref. 81 . 


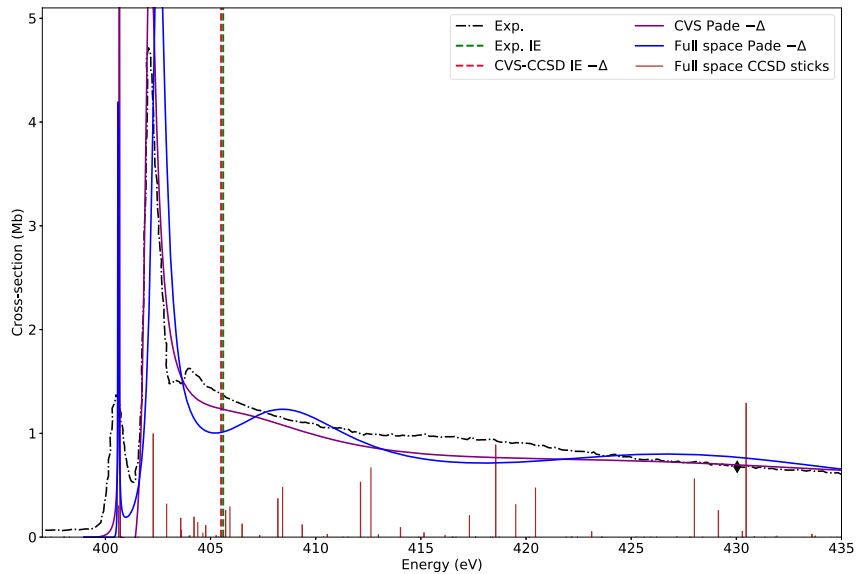

(a)

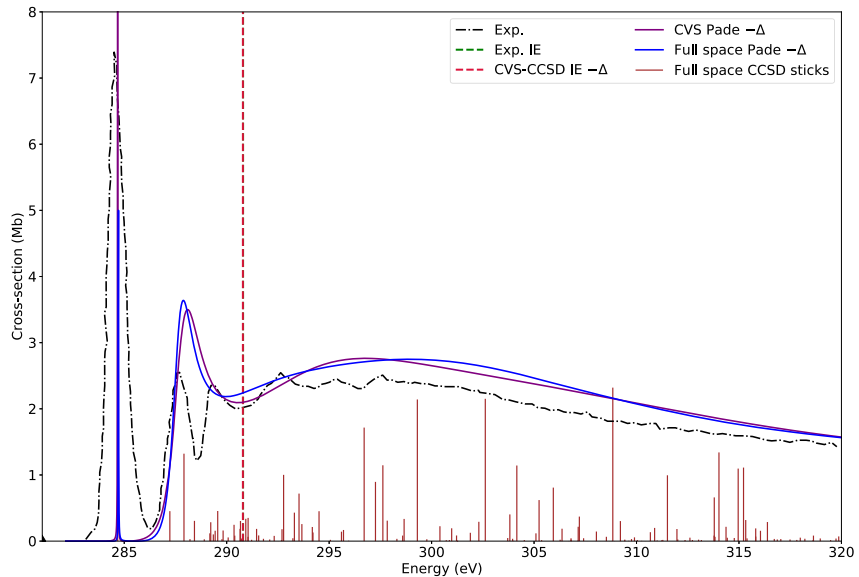

(b)

FIG. 7. Full-space CCSD vs CVS-CCSD cross sections. The full space calculation was performed with $J=3000$ in the same basis set and geometry used in Fig. 6 (a) (ammonia) and Fig. 1(a) (ethylene). The same values of the shift $\Delta=1.02 \mathrm{eV}$ (ammonia) and $\Delta=0.83 \mathrm{eV}$ (ethylene) were applied to both cross sections. The experimental results are from Refs. 77 and 81.

one explicitly leaves out all excitations below the first core excited one.

\section{F. Acetaldehyde}

The oxygen and carbon K-shell cross sections of acetaldehyde are presented in Figs. 8 and 9, respectively, together with the corresponding experimental data. ${ }^{83}$ They were computed at the CVS-CCSD and CVS-CC2 levels using the aug-cc-pVTZ basis on the $\mathrm{O}$ and $\mathrm{C}$ atoms, the aug-cc-pVDZ on the $\mathrm{H}$ atoms, and with the $(7 \mathrm{~s} 7 \mathrm{p} 7 \mathrm{~d})_{n=2-8}$ continuumlike set $^{73}$ in the center of mass. The Lanczos chain length was truncated at $J=250$.

Experimental results for acetaldehyde have been reported by Hitchcock and Brion ${ }^{83}$ using the EELS technique and by Prince et al. ${ }^{82}$ using synchrotron radiation. The high resolution experimental spectrum ${ }^{82}$ obtained at the oxygen K-edge shows an intense peak centered at $531.5 \mathrm{eV}$, assigned to a $1 \mathrm{~s}_{\mathrm{O}} \rightarrow \pi^{*}$ transition, followed by two weaker transitions to the $3 \mathrm{~s}$ and $3 \mathrm{p}$ states centered at 535.4 and $536.3 \mathrm{eV}$, respectively. Theoretical core excitation energies of acetaldehyde were recently obtained by Myhre, Coriani, and Koch ${ }^{20}$

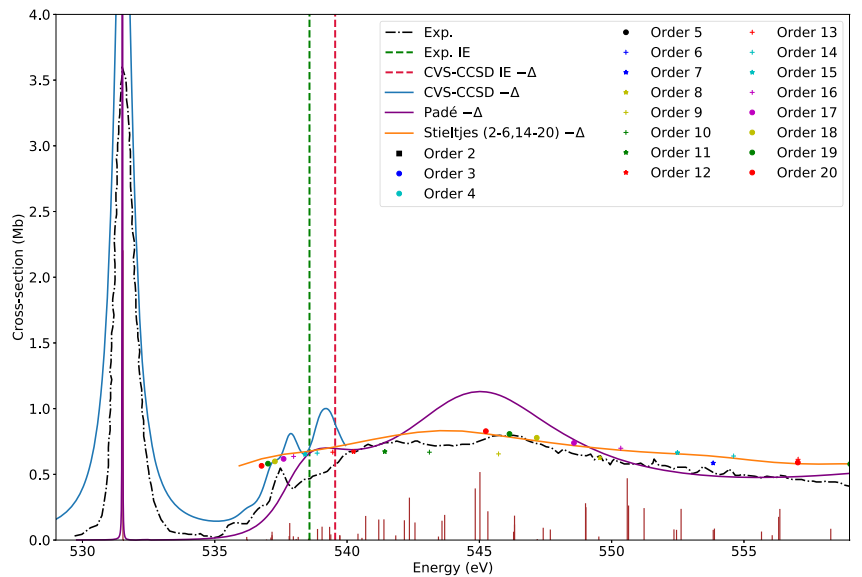

(a)

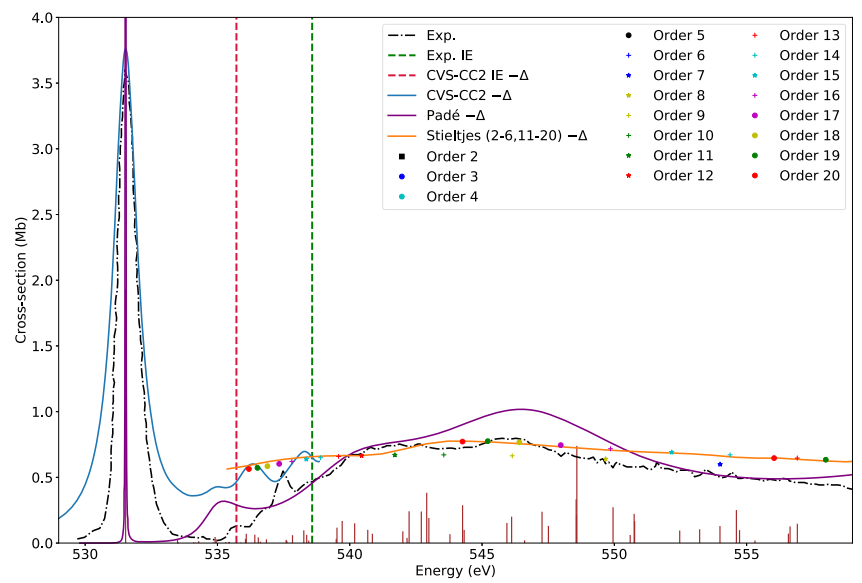

(b)

FIG. 8. Acetaldehyde. CVS-CCSD [panel (a)] and CVS-CC2 [panel (b)] oxygen K-edge cross sections obtained with $J=250$ and the aug-cc-pVTZ(C,O)/aug-cc$\mathrm{pVDZ}(\mathrm{H})+(7 \mathrm{~s} 7 \mathrm{p} 7 \mathrm{~d})_{n=2-8}$ basis set. The discrete peaks were broadened with a Lorentzian function, HWHM $=0.41 \mathrm{eV}$ [panel (a)] and $0.54 \mathrm{eV}$ [panel (b)]. The calculated spectra are shifted by $\Delta=0.84 \mathrm{eV}$ [panel (a)] and $\Delta=0.82 \mathrm{eV}$ [panel (b)]. Experimental results are from Ref. 82. 


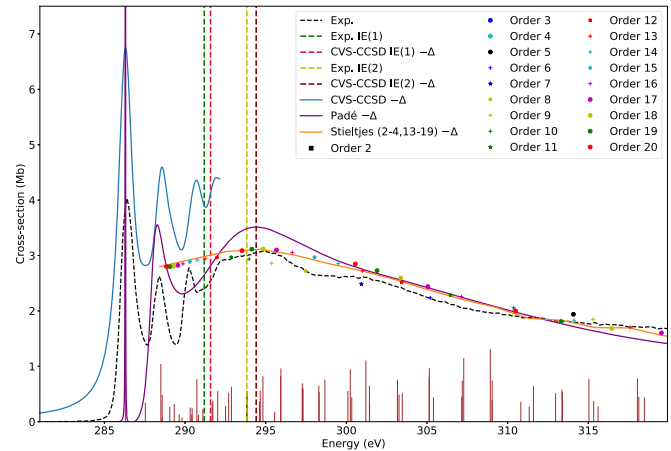

(a)

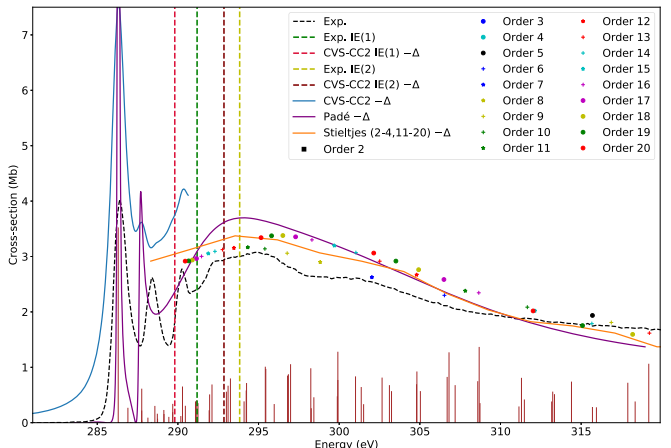

(b)

FIG. 9. Acetaldehyde. CVS-CCSD (a) and CVS-CC2 (b) carbon K edge cross sections obtained with $J=250$ and the aug-cc-pVTZ (C,O)/aug-cc-pVDZ(H)+(7s7p7d) $n=2-8$ basis set. The discrete peaks were broadened with a Lorentzian function, HWHM $=0.54 \mathrm{eV}$ [panels (a) and (b)]. The calculated spectra are shifted by $\Delta=0.62 \mathrm{eV}$ [panel (a)] and $\Delta=2.13 \mathrm{eV}$ [panel (b)]. Experimental results were taken from Refs. 75 and 83.

applying CVS-CCSD and multilevel (ML-)CCSD with the aug-ccpCVDZ and aug-cc-p(C)VDZ basis sets, respectively. The authors calculated the $1 \mathrm{~s}_{\mathrm{O}} \rightarrow \pi^{*}$ transition energy at $534.65 \mathrm{eV}$. Our CVS(Lanczos)CCSD transition energy in the aug-cc-pVTZ(C,O)/augcc-pVDZ $(\mathrm{H})+(7 \mathrm{~s} 7 \mathrm{p} 7 \mathrm{~d})_{n=2-8}$ basis set is $532.3 \mathrm{eV}$ for the $1 \mathrm{~s}_{\mathrm{O}} \rightarrow \pi^{*}$ transition, with an energy difference of only $\Delta=0.8 \mathrm{eV}$ from the experimental $1 \mathrm{so} \rightarrow \pi^{*}$ band. The shifted CVS-CCSD IE remains $1 \mathrm{eV}$ higher than the experimental value, while the shifted CC2 IE is $3 \mathrm{eV}$ lower than the experiment. As it can be observed from Fig. 8(a), at the CVS-CCSD level both continuum procedures describe the continuum tail in reasonable agreement with the experiment. The Padé treatment gives rise to a relatively high and broad band around $545 \mathrm{eV}$ not observed in the Stieltjes imaging spectrum which is all in better agreement with the experiment. This same behavior is observed for the Padé and Stieltjes photoionization cross sections [Fig. 8(b)] obtained at the CVS-CC2 level of calculation.

The carbon K-shell cross sections computed at the CVS-CCSD and CVS-CC2 levels are shown in Figs. 9(a) and 9(b), respectively. The experimental curve ${ }^{83}$ shows three well resolved peaks centered at $286.2 \mathrm{eV}, 288.4 \mathrm{eV}$, and $290.2 \mathrm{eV}$, which have been assigned to $1 \mathrm{~s}_{\mathrm{C}} \rightarrow \pi^{*}(\mathrm{C}=\mathrm{O}), 1 \mathrm{~s}_{\mathrm{C}} \rightarrow \pi^{*}\left(\mathrm{CH}_{3}\right)$, and $1 \mathrm{~s}_{\mathrm{C}} \rightarrow \pi^{*}\left(\mathrm{CH}_{3}\right)$ transitions, respectively. ${ }^{83}$ The CVS-CCSD stick spectrum broadened with Lorentzian functions with $\mathrm{HWHM}=0.54 \mathrm{eV}$ shown in Fig. 9(a) describes the three main peaks of the experimental spectrum, once a shift of $-0.62 \mathrm{eV}$ is applied to the energy axis. As before, the Padé analytic continuation procedure gives rise to only the first two peaks corresponding to the $1 \mathrm{~s}_{\mathrm{C}} \rightarrow \pi^{*}(\mathrm{C}=\mathrm{O})$ and the $1 \mathrm{~s}_{\mathrm{C}} \rightarrow \pi^{*}\left(\mathrm{CH}_{3}\right)$ transitions. From Fig. 9, one observes that the shifted $1 s_{C}^{-1}$ IEs calculated with CVS-CCSD for the carbon atoms are in better agreement with the experimental values than the corresponding CVS-CC2 results.

Nevertheless, the spectral features of the photoionization cross sections obtained with the Pade and the Stieltjes procedures show a reasonable agreement with the experiment. The CVS-CC2 results are less accurate than the CVS-CCSD ones in the discrete region, but above the threshold, the difference between them is smaller and both levels of calculation describe the spectral features in reasonable agreement with experiments.

\section{G. Pyrrole}

The nitrogen and carbon $\mathrm{K}$ shell photoionization cross sections of pyrrole are shown in Figs. 10 and 11, together with experimental data. ${ }^{84}$ They were obtained at the CVS-CCSD and CVS-CC2 levels using the aug-cc-pVTZ basis on the nitrogen and carbon atoms, the aug-cc-pVDZ on the hydrogen atoms, and the $(7 \mathrm{~s} 7 \mathrm{p} 7 \mathrm{~d})_{n=2-8}{ }^{73}$ continuumlike set placed at the center of mass of the molecule. The Lanczos chain length was truncated at $J=250$. Since the experimental data ${ }^{84}$ were originally reported in arbitrary units, the experimental values were scaled in a way that the continuum tail of the experimental photoionization cross section matched the theoretical ones.

In Fig. 10, the pyrrole inner-shell EELS ${ }^{84}$ data for $\mathrm{N}$ show an intense and narrow peak centered at $402.3 \mathrm{eV}$ due to a N $1 \mathrm{~s} \rightarrow \pi^{*}$ transition, followed by a $1 \mathrm{~s}_{\mathrm{N}} \rightarrow 4 \mathrm{p}$ transition at $405.9 \mathrm{eV}$. Above the ionization threshold (406.1 eV), a broad peak centered at $406.7 \mathrm{eV}$ assigned to $1 \mathrm{~s}_{\mathrm{N}} \rightarrow \sigma^{*}(\mathrm{C}-\mathrm{N})$ transitions is followed by the weaker transition $1 \mathrm{~s}_{\mathrm{N}} \rightarrow \sigma^{*}(\mathrm{C}-\mathrm{C})$ near $413.0 \mathrm{eV}$ extending to a continuum tail up to $440 \mathrm{eV}$. The transition energies of the $1 \mathrm{~s}_{\mathrm{N}} \rightarrow \pi^{*}$ state computed at the CVS-CCSD and CVS-CC2 levels are 1.17 and $1.46 \mathrm{eV}$, respectively, above the corresponding experimental value. The computed cross sections shown in Fig. 10 were shifted correspondingly. The shifted $1 \mathrm{~s}_{\mathrm{N}}^{-1}$ IE obtained at the CVS-CCSD and CVS-CC2 levels are represented by the red dashed lines in Fig. 10. Once again, agreement between the shifted CVS-CCSD IE and experiment is better than for CVS-CC2 counterpart, as can be appreciated from the results in Fig. 10. The high energy tail of the photoionization cross sections follows closely the experimental tail at both CVS-CCSD and CVS-CC2 levels. The Padé procedure at both CC levels yields a broad band above the IE threshold at $406.7 \mathrm{eV}$ (which has been assigned to a $1 \mathrm{~s}_{\mathrm{N}} \rightarrow \sigma^{*}(\mathrm{C}-\mathrm{N})$ transition), though with much higher intensity than the experimental, as well as a slight increase at around $415 \mathrm{eV}$. 


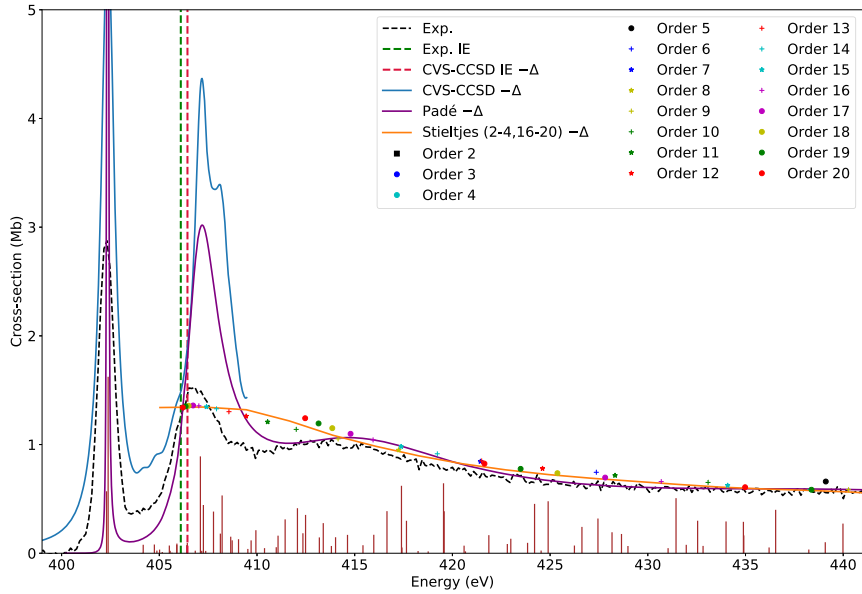

(a)

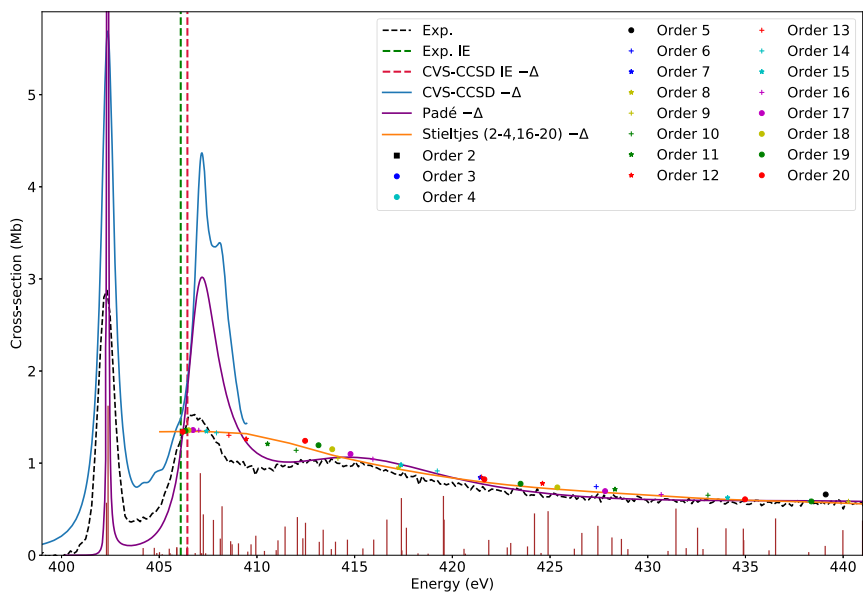

(b)

FIG. 10. Pyrrole. CVS-CCSD [panel (a)] and CVS-CC2 [panel (b)] nitrogen K-edge cross sections obtained with $J=250$ and the aug-cc-pVTZ (C,N)/aug-cCpVDZ $(\mathrm{H})+(7 \mathrm{~s} 7 \mathrm{p} 7 \mathrm{~d})_{n=2-8}$ basis set. The discrete peaks were broadened with a Lorentzian function, HWHM $=0.41 \mathrm{eV}$. The calculated spectra were shifted by $\Delta=1.17$ $\mathrm{eV}$ [panel (a)] and $\Delta=1.40 \mathrm{eV}$ [panel (b)]. Experimental results are from Refs. 75 and 84.

The carbon K-shell EELS ${ }^{84}$ experimental spectrum is shown in Fig. 11 along with the results of our calculations. The experiment presents an intense and narrow peak centered at $285.6 \mathrm{eV}$ attributed to a $1 \mathrm{~s}_{\mathrm{C}} \rightarrow \pi^{*}$ transition. Above the $1 \mathrm{~s}_{\mathrm{C}}^{-1}$ ionization thresholds $[289.8 \mathrm{eV}(\mathrm{C}-\mathrm{C})$ and $290.8 \mathrm{eV}(\mathrm{C}-\mathrm{N})]$, there are two main broad peaks, the first due to the $1 \mathrm{~s}_{\mathrm{C}} \rightarrow \sigma^{*}(\mathrm{C}-\mathrm{N})$ transition centered at $291.3 \mathrm{eV}$ and the second one to the $1 \mathrm{~s}_{\mathrm{C}} \rightarrow \sigma^{*}(\mathrm{C}-\mathrm{C})$ transition at $296.8 \mathrm{eV}$, followed by a continuum tail which extends up to $330 \mathrm{eV}$. The C $1 \mathrm{~s} \rightarrow \pi^{*}$ transition energy calculated at the CVS-CCSD and
CVS-CC2 levels is higher than the experimental value by 1.91 and $3.30 \mathrm{eV}$, respectively. Once again, the shifted carbon K-edge CVSCCSD ionization energies, represented by dashed lines in Fig. 11, are in better agreement with the experimental values than the CVS$\mathrm{CC} 2$ results. The continuum part of both spectra show good agreement with the experimental continuum tail profile. The CVS-CC2 photoabsorption cross sections [Fig. 11(b)] look very similar to the CVS-CCSD ones at both the discrete and the continuum regions of the spectra.

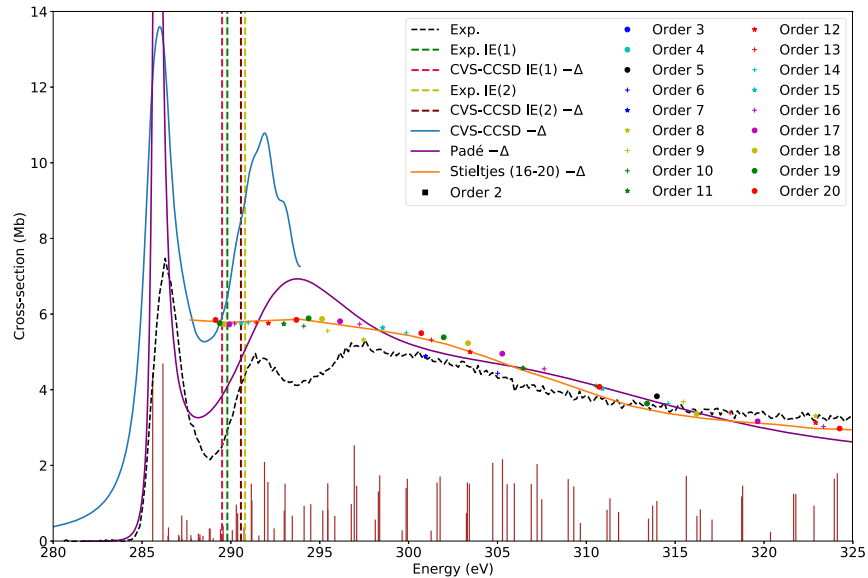

(a)

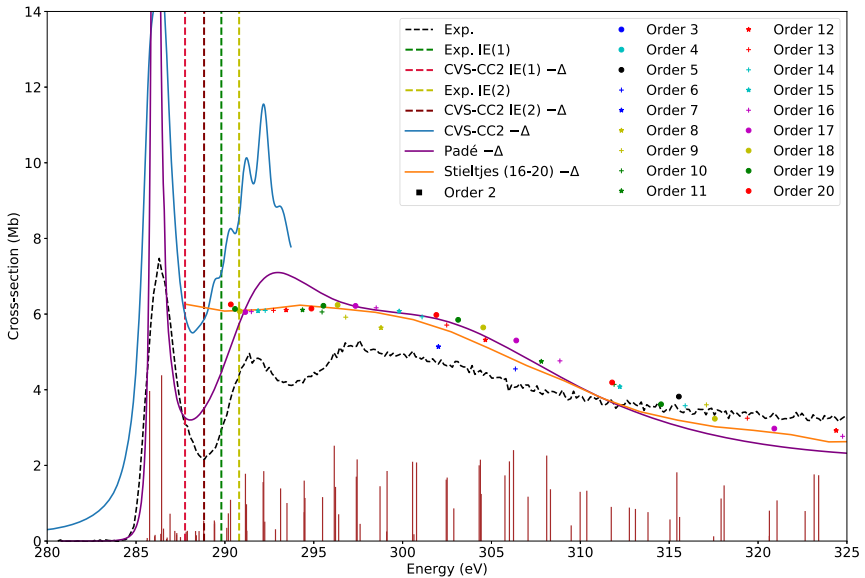

(b)

FIG. 11. Pyrrole. CVS-CCSD [panel (a)] and CVS-CC2 [panel (b)] carbon $K$ shell cross sections obtained with $J=250$ with the aug-cc-pVTZ (C,N)/aug-ccpVDZ $(\mathrm{H})+(7 \mathrm{~s} 7 \mathrm{p} 7 \mathrm{~d})_{n=2-8}$ basis set. The discrete peaks were broadened with a Lorentzian function, HWHM $=0.68 \mathrm{eV}$ [panel (a)] and $0.54 \mathrm{eV}$ [panel (b)]. The CVS-CCSD and CVS-CC2 spectra were shifted by $\Delta=1.41 \mathrm{eV}$ and $\Delta=2.48 \mathrm{eV}$, respectively. Experimental results taken from Refs. 75 and 84. 
We also performed CVS-CCSD calculations using the continuumlike set employed in Ref. 61 where the photoionization cross section of pyrrole was obtained at the TDDFT/PBE0 level with the d-aug-cc-pVTZ atomic basis set complemented with a specifically tailored $(5 \mathrm{~s} 5 \mathrm{p} 4 \mathrm{~d})$ continuumlike set placed at the center of mass of the system. The CVS-CCSD results are presented in Fig. 12 along with the experimental values. Figure 12(a) shows the nitrogen Kedge photoionization cross section, and Fig. 12(b) shows the carbon $\mathrm{K}$-edge results, all of them obtained with the aug-cc-pVTZ basis on $\mathrm{N}$ and $\mathrm{C}$, the aug-cc-pVDZ on $\mathrm{H}$, and the (5s5p4d) continuum set as defined in Ref. 61. Considering the first results for nitrogen, it can be seen that the cross section generated by the Stieltjes imaging procedure is very similar to the one obtained with the $(7 \mathrm{~s} 7 \mathrm{p} 7 \mathrm{~d})_{n=2-8}$ set of Kaufmann, Baumeister, and Jungen [see Fig. 10(a)]. The major difference between the two continuum sets is observed when using the Padé analytic continuation procedure. Comparing the corresponding Padé cross sections of Figs. 10(a) and 12(a), one can notice the presence of a strong band near $418 \mathrm{eV}$ when the $5 \mathrm{~s}, 5 \mathrm{p}, 4 \mathrm{~d}$ continuum set is used. The pyrrole results in Ref. 61 do not look so bad because the larger and more diffuse d-aug-cc-pVTZ basis set somehow compensated the poor description of the continuum provided by the $(5 \mathrm{~s} 5 \mathrm{p} 4 \mathrm{~d})$ set. This also shows that it is possible to reduce the size of the atomic basis set as long as a proper continuumlike set is used. At the $\mathrm{C} \mathrm{K}$-edge, on the other hand, the photoionization cross sections obtained with the $5 \mathrm{~s}, 5 \mathrm{p}, 4 \mathrm{~d}$ continuum set are very similar to those obtained with Kaufmann's $(7 \mathrm{~s} 7 \mathrm{p} 7 \mathrm{~d})_{n=2, \ldots, 8}$ continuumlike set.

\section{H. Furan}

The computed oxygen and carbon K-edge cross sections of furan are presented in Figs. 13 and 14 together with their experimental counterparts. ${ }^{84}$ The calculations were performed using the
aug-cc-pVTZ basis on the $\mathrm{O}$ and $\mathrm{C}$ atoms, the aug-cc-pVDZ basis on the $\mathrm{H}$ atoms, and the set $(7 \mathrm{~s} 7 \mathrm{p} 7 \mathrm{~d})_{n=2-8}{ }^{73}$ of continuumlike functions placed in the center of mass of the system. The Lanczos chain length was truncated at $J=250$. The experimental values for furan are reported in arbitrary units and are scaled such that the continuum tail of the experimental photoionization cross section matches the theoretical one.

The oxygen K-edge photoabsorption spectrum $^{84}$ of furan presents an intense and narrow band centered at $535.3 \mathrm{eV}$ assigned to a $1 \mathrm{~s}_{\mathrm{O}} \rightarrow \pi^{*}$ transition, followed by a broader and less intense band centered at $539.4 \mathrm{eV}$ attributed to $1 \mathrm{~s}_{\mathrm{O}} \rightarrow \sigma^{*}(\mathrm{C}-\mathrm{O})$ transitions. Above the $1 \mathrm{~s}_{\mathrm{O}}^{-1} \mathrm{IE}$, a broad peak centered at $545.3 \mathrm{eV}$ has been assigned to the $1 \mathrm{~s}_{\mathrm{O}} \rightarrow \sigma^{*}(\mathrm{C}-\mathrm{C})$ transition. ${ }^{84}$ Duflot et al. ${ }^{85}$ reported theoretical results for the furan $1 \mathrm{~s}_{\mathrm{O}} \rightarrow \pi^{*}$ and $1 \mathrm{~s}_{\mathrm{O}} \rightarrow \sigma^{*}$ transition energies at $535.6 \mathrm{eV}$ and $540.0 \mathrm{eV}$, respectively, obtained from configuration interactions singles (CIS) calculations using core-relaxed molecular orbitals with the TZP basis set supplemented with a set of Rydberg orbitals ( $3 \mathrm{~s} 3 \mathrm{p} 3 \mathrm{~d} 4 \mathrm{~s} 4 \mathrm{p}$ ) on the core-excited atom. Our excitation energies for the $1 \mathrm{~s}_{\mathrm{O}} \rightarrow \pi^{*}$ transition at the CVS-CCSD and CVS-CC2 levels are 1.54 and $0.45 \mathrm{eV}$ above the corresponding experimental value, respectively. The $1 \mathrm{~s}_{\mathrm{O}}^{-1}$ IEs calculated at the CVSCCSD and CVS-CC2 levels are represented by the red dashed lines in Fig. 13, shifted by the same amount used to shift the NEXAFS spectrum. As for the previous molecules and $\mathrm{O}$ edges, the agreement between the shifted CVS-CCSD IE and the experimental value is much better than for the CVS-CC2 result, as can be clearly seen from Fig. 13.

Concerning the CVS-CCSD oxygen K-shell photoionization cross sections in Fig. 13(a), we observe that the Padé cross section overestimates the $1 \mathrm{~s}_{\mathrm{O}} \rightarrow \sigma^{*}(\mathrm{C}-\mathrm{O})$ peak at around $540 \mathrm{eV}$, whereas the broad feature at $\approx 545 \mathrm{eV}$ in the experimental spectrum appears to be shifted toward higher energy $(\approx 550 \mathrm{eV})$. This feature is absent in the Stieltjes imaging photoionization cross section. The CVS-CC2

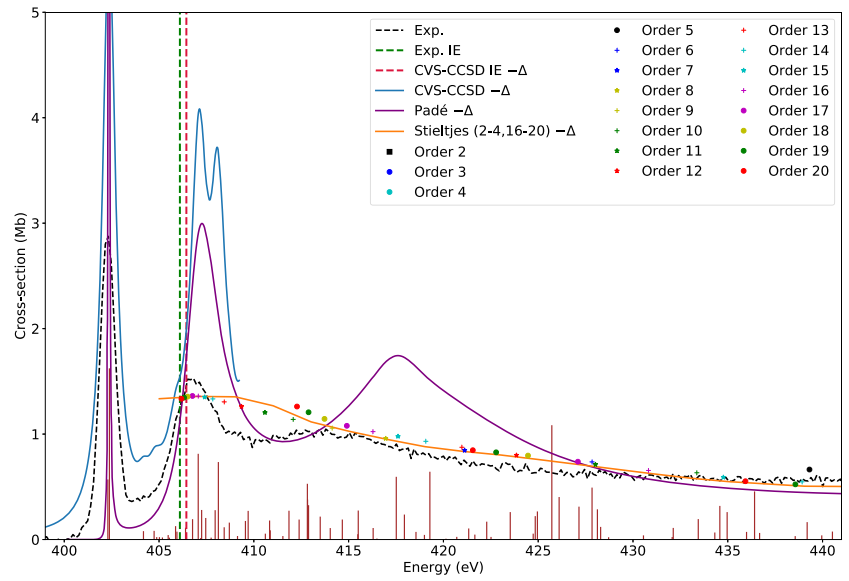

(a)

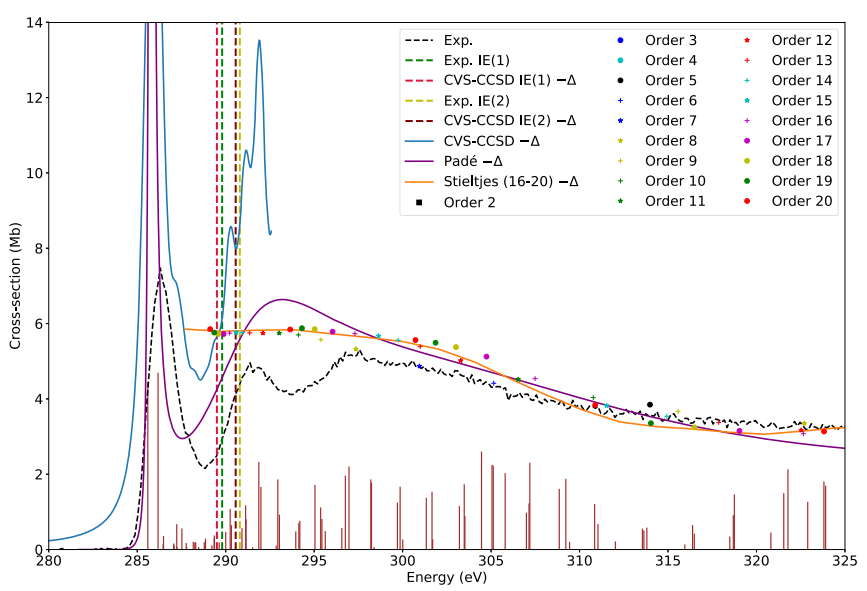

(b)

FIG. 12. Pyrrole. CVS-CCSD nitrogen (a) and carbon (b) K-edge cross sections obtained with $J=250$ and the aug-cc-pVTZ(C,N)/aug-cc-pVDZ(H) basis set. The basis set used to represent the continuum region, placed at the center of mass of the molecule, was the $(5 \mathrm{~s} 5 \mathrm{p} 4 \mathrm{~d})$ continuumlike set employed in Ref. 61 . The discrete peaks were broadened with a Lorentzian function, HWHM $=0.41 \mathrm{eV}$. The calculated spectra are shifted by $\Delta=1.17 \mathrm{eV}$ [panel (a)] and $\Delta=1.41 \mathrm{eV}$ [panel (b)]. Experimental results are from Refs. 75 and 84. (a) CVS-CCSD, $1 \mathrm{~s}_{\mathrm{N}}, 5 s, 5 p, 4 d$; (b) CVS-CCSD, $1 \mathrm{~s}_{\mathrm{C}}, 5 s, 5 p, 4 d$. 


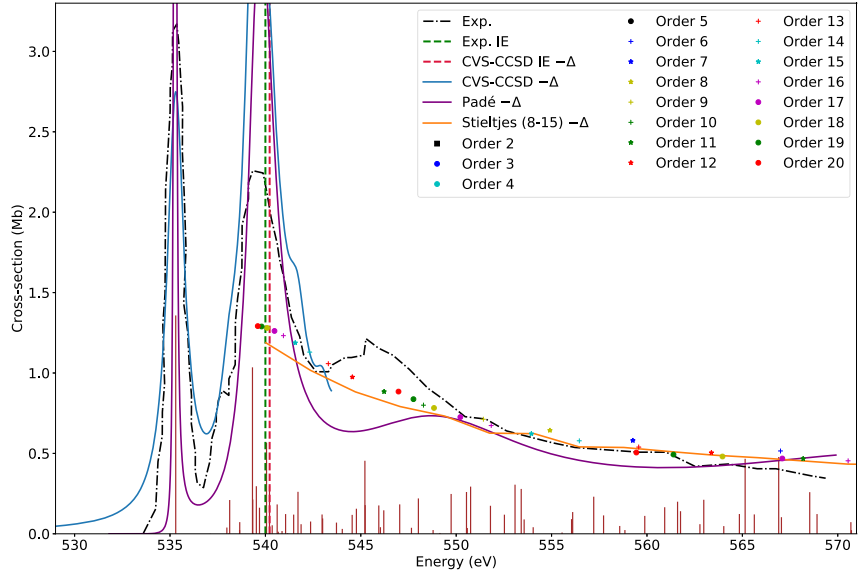

(a)

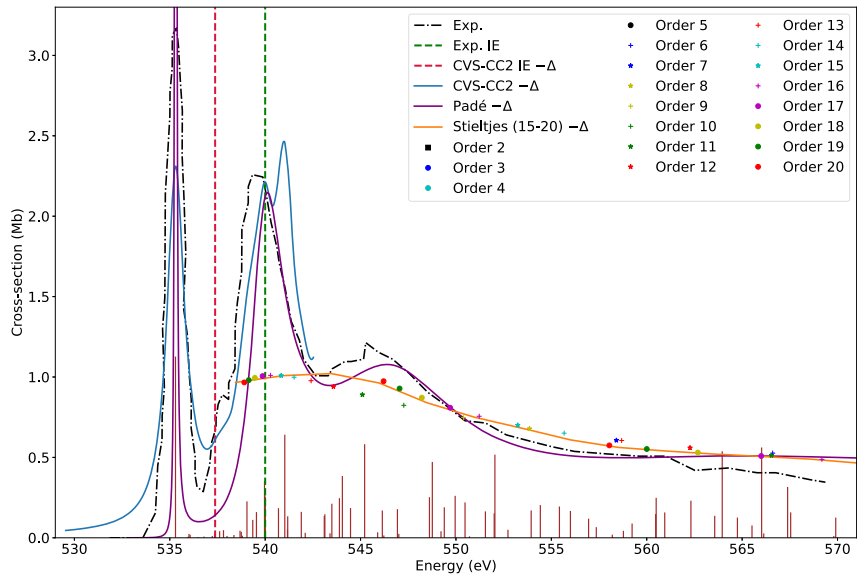

(b)

FIG. 13. Furan. CVS-CCSD (a) and CVS-CC2 (b) oxygen K-edge cross sections obtained with $J=250$ and the aug-cC-pVTZ $(C, 0) /$ aug-cc-pVDZ(H)+(7s7p7d) $n=2-8$ basis set. The discrete peaks were broadened with a Lorentzian function, HWHM $=0.54 \mathrm{eV}$. The calculated spectra were shifted by $\Delta=1.54 \mathrm{eV}[\mathrm{panel}(\mathrm{a})]$ and $\Delta=0.45 \mathrm{eV}[\mathrm{panel}$ (b)]. Experimental results are from Ref. 84.

Padé cross section [see Fig. 13(b)] presents both the broad band near $540 \mathrm{eV}$ and the one at $545 \mathrm{eV}$, and the continuum profile in better agreement with the experimental photoionization cross section. The CVS-CC2 results are in line with the TDDFT/PBE0 results presented in Ref. 61.

The computed carbon K-edge photoionization cross sections and the experimental data ${ }^{84}$ of furan are shown in Fig. 14. In the experimental spectrum, there are four peaks, centered at 285.6, $286.5,288.5$, and $289.3 \mathrm{eV}$, attributed to $1 \mathrm{~s}_{\mathrm{C}} \rightarrow \pi^{*}$ transitions. ${ }^{84}$ Two broad and intense peaks, above the $1 \mathrm{~s}_{\mathrm{C}}$ ionization threshold, centered at 291.4 and $297.0 \mathrm{eV}$ have been assigned to the $1 \mathrm{~s}_{\mathrm{C}} \rightarrow$ $\sigma^{*}(\mathrm{C}-\mathrm{O})$ and $1 \mathrm{~s}_{\mathrm{C}} \rightarrow \sigma^{*}(\mathrm{C}-\mathrm{C})$ transitions, respectively. ${ }^{84}$ The first transition to a $1 \mathrm{~s}_{\mathrm{C}} \rightarrow \pi^{*}$ state calculated at the CVS-CCSD and CC2 levels lies 0.5 and $1.8 \mathrm{eV}$ above the experimental value, respectively. The results obtained at the CVS-CCSD level shown in Fig. 14(a) are in reasonable agreement with the experimental cross section. The region below the thresholds is well described by the stick spectrum broadened with Lorentzian functions with $\mathrm{HWHM}=0.54 \mathrm{eV}$, and the continuum tail generated by Stieltjes imaging is also in good agreement with the experimental profile. The CVS-CC2 photoionization cross sections obtained from Stieltjes imaging and from the analytic continuation procedure are shown in Fig. 14(b) where one can see that the results are qualitatively similar to those obtained at the CVS-CCSD level. The theoretical profile of the discrete and

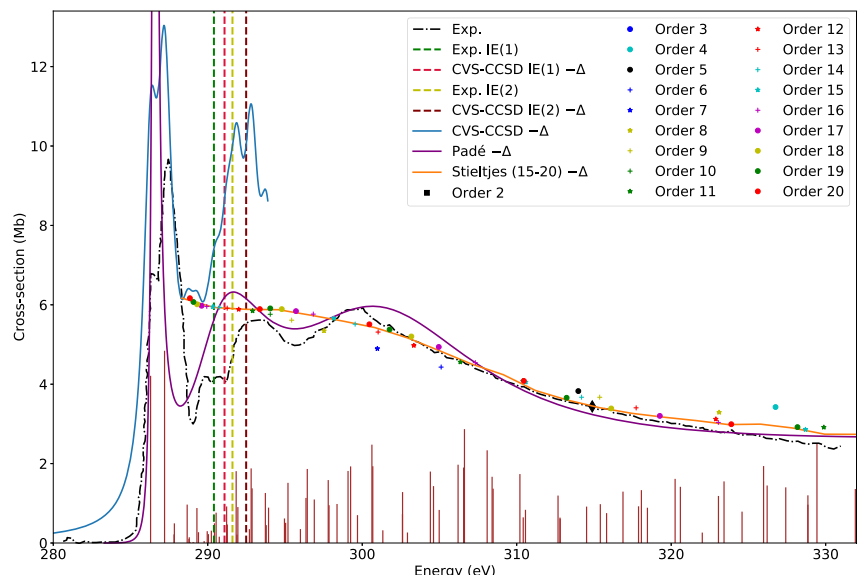

(a)

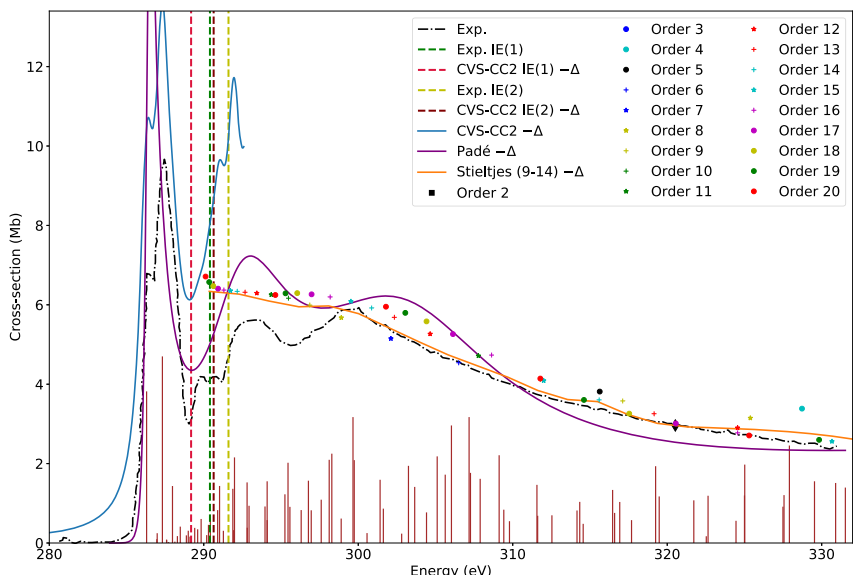

(b)

FIG. 14. Furan. CVS-CCSD (a) and CVS-CC2 (b) carbon K-edge cross sections obtained with $J=250$ in the aug-cc-pVTZ(C,0)/aug-cc-pVDZ(H)+(7s7p7d) $n=2-8$ basis set. The discrete peaks were broadened with a Lorentzian function, $\mathrm{HWHM}=0.54 \mathrm{eV}$ [panels (a) and (b)]. The calculated spectra were shifted by $\Delta=0.51 \mathrm{eV}[$ panel (a)] and $\Delta=1.81 \mathrm{eV}$ [panel (b)]. Experimental results from Ref. 84 . 
continuum parts of the spectra shown in Fig. 14 agree with the results obtained at the TDDFT/PBE0 level reported in Ref. 61.

\section{CONCLUSIONS}

In Ref. 61, we reported total photoionization cross sections in the $\mathrm{X}$-ray region of the spectrum at the oxygen, carbon, and nitrogen $\mathrm{K}$-edges for a number of molecules, using an analytical continuation technique based on the Padé approximants and pseudospectra from CC and TDDFT calculations At the CC level, however, the investigation was limited to quite small systems, namely, water and ammonia, since very large Lanczos chain lengths were required to access the core excited states, restricting the study of the larger molecules to the TDDFT level.

In this paper, we show that reliable photoabsorption cross sections, below and above the K-edge core ionization thresholds, can be obtained for larger molecules, using a core-valence-separated CC linear-response scheme based on the asymmetric Lanczos algorithm and either the analytic continuation procedure or the Stieltjes imaging procedure. We compared the results obtained at the oxygen, nitrogen, and carbon K-edges for a number of small and medium sized molecules (water, ammonia, ethylene, carbon dioxide, acetaldehyde, furan, and pyrrole) using a larger basis set $(7 \mathrm{~s} 7 \mathrm{p} 7 \mathrm{~d})_{n=2-8}$ to simulate the continuum region of the spectrum than the one previously used. ${ }^{61}$ The results obtained with both the Padé analytic continuation procedure and the Stieltjes imaging technique indicate that, generally speaking, the cross sections agree with each other and are in good agreement with the available experimental data. We also showed that for larger molecules, the size of the atomic basis set can be reduced as long as a larger continuumlike basis set is used, as exemplified for the pyrrole molecule, opening the possibility of treating large molecules at the CC level of theory. For pyrrole, we used both the $(7 \mathrm{~s} 7 \mathrm{p} 7 \mathrm{~d})_{n=2-8}$ and the ad hoc derived ${ }^{6}$ $(5 \mathrm{~s}, 5 \mathrm{p}, 4 \mathrm{~d})$ continuum sets with a smaller atomic orbital set, which led to good results for the larger $(7 \mathrm{~s} 7 \mathrm{p} 7 \mathrm{~d})_{n=2-8}$ continuum set and poor agreement when using the smaller $(5 \mathrm{~s}, 5 \mathrm{p}, 4 \mathrm{~d})$ continuum set. The TDDFT results presented in Ref. 61 for pyrrole did not look so bad because the larger and more diffuse d-aug-cc-pVTZ basis set somehow compensated for the poorer description of the continuum by the $(5 \mathrm{~s}, 5 \mathrm{p}, 4 \mathrm{~d})$ continuum set, showing that it is possible to reduce the atomic basis set as long as a proper continuumlike set is used. Overall, the present results show that the Stieltjes and the Padé analytic continuation procedures in conjunction with a corevalence separated CCLR scheme based on the asymmetric Lanczos algorithm yield nicely converged photoionization cross sections at the oxygen, carbon, and nitrogen edges of organic molecules, with the advantage of compromising accuracy and computational cost of the CVS-CCLR scheme.

The results presented also serve as a validation step in the generalization of our protocols to core-level photoionization cross sections of transient valence-excited states, which are of interest for the simulation of time-resolved X-ray spectroscopy. ${ }^{6}$

\section{ACKNOWLEDGMENTS}

T.M. and S.C. acknowledge support from the European Union's Horizon 2020 Research and Innovation Programme under the
Marie Skłodowska-Curie Grant Agreement No. 765739, "COSINEEuropean Training Network on COmputational Spectroscopy In Natural sciences and Engineering." S.C. also acknowledges funding from the Independent Research Fund Denmark, DFFForskningsprojekt2 Grant No. 7014-00258B.

B.N.C.T., M.A.C.N., and A.B.R. acknowledge Conselho Nacional de Desenvolvimento Científico e Tecnológico (CNPq), Coordenação de Aperfeiçoamento Pessoal de Nível Superior (CAPES) - Finance Code 001, and Fundação de Amparo à Pesquisa do Estado do Rio de Janeiro (FAPERJ) for financial support.

\section{REFERENCES}

${ }^{1}$ D. Egorov, L. Schwob, M. Lalande, R. Hoekstra, and T. Schlatholter, Phys. Chem. Chem. Phys. 18, 26213 (2016).

${ }^{2}$ O. González-Magaña, G. Reitsma, M. Tiemens, L. Boschman, R. Hoekstra, and T. Schlathölter, J. Phys. Chem. A 116, 10745 (2012).

${ }^{3}$ I. Ljubić, A. Kivimäki, and M. Coreno, Phys. Chem. Chem. Phys. 18, 10207 (2016).

${ }^{4}$ J. Ontaneda, R. E. J. Nicklin, A. Cornish, A. Roldan, R. Grau-Crespo, and G. Held, J. Phys. Chem. C 120, 27490 (2016)

${ }^{5}$ O. Endo, M. Nakamura, K. Amemiya, and H. Ozaki, Langmuir 33, 3934 (2017).

${ }^{6}$ A. G. Thomas, M. J. Jackman, M. Wagstaffe, H. Radtke, K. Syres, J. Adell, A. Lévy, and N. Martsinovich, Langmuir 30, 12306 (2014).

${ }^{7}$ M. Di Giovannantonio, M. El Garah, J. Lipton-Duffin, V. Meunier, L. Cardenas, Y. Fagot Revurat, A. Cossaro, A. Verdini, D. F. Perepichka, F. Rosei, and G. Contini, ACS Nano 7, 8190 (2013).

${ }^{8}$ G. W. Wang, Y. Ma, X. N. Song, S. Z. Jiang, W. W. Yue, C. K. Wang, and Y. Luo, J. Phys. Chem. C 120, 13779 (2016).

${ }^{9}$ X.-N. Song, G.-W. Wang, Y. Ma, S.-Z. Jiang, W.-W. Yue, C.-K. Wang, and Y. Luo, J. Phys. Chem. A 120, 9932 (2016).

${ }^{10}$ D. Erbahar, T. Susi, X. Rocquefelte, C. Bittencourt, M. Scardamaglia, P. Blaha, P. Guttmann, G. Rotas, N. Tagmatarchis, X. Zhu, A. P. Hitchcock, and C. P. Ewels, Sci. Rep. 6, 35605 (2016).

${ }^{11}$ T. Wolf, R. Myhre, J. Cryan, S. Coriani, R. Squibb, A. Battistoni, N. Berrah, C. Bostedt, P. Bucksbaum, G. Coslovich, R. Feifel, K. Gaffney, J. Grilj, T. Martinez, S. Miyabe, S. Moeller, M. Mucke, A. Natan, R. Obaid, T. Osipov, O. Plekan, S. Wang, H. Koch, and M. Gühr, Nat. Commun. 8(1), 29 (2017).

${ }^{12}$ P. M. Kraus, M. Zürch, S. K. Cushing, D. M. Neumark, and S. R. Leone, Nat. Rev. Chem. 2, 82 (2018).

${ }^{13} X$-Ray Absorption and X-ray Emission Spectroscopy; Theory and Applications, edited by J. van Bokhoven and C. Lamberti (Wiley \& Sons, 2016).

${ }^{14}$ Synchrotron Radiation: Basics, Methods and Applications, edited by S. Mobilio, F. Boscherini, and C. Meneghini (Springer, 2014).

${ }^{15}$ X-Ray Free Electron Lasers: Applications in Materials, Chemistry and Biology, Energy and Environment Series No. 18, edited by U. Bergmann, V. Yachandra, and J. Yano (Royal Society of Chemistry, 2017).

${ }^{16}$ C. Milne, T. Penfold, and M. Chergui, Coord. Chem. Rev. 277-278, 44 (2014).

${ }^{17}$ A. B. Rocha, J. Chem. Phys. 134, 024107 (2011).

${ }^{18}$ B. N. Tenório, C. E. de Moura, R. R. Oliveira, and A. B. Rocha, Chem. Phys. 508, 26 (2018).

${ }^{19}$ C. E. V. de Moura, R. R. Oliveira, and A. B. Rocha, J. Mol. Model. 19, 2027 (2013).

${ }^{20}$ R. H. Myhre, S. Coriani, and H. Koch, J. Chem. Theory Comp. 12, 2633 (2016).

${ }^{21}$ I. Corral, J. González-Vázquez, and F. Martín, J. Chem. Theory Comp. 13, 1723 (2017).

${ }^{22}$ H. Ågren and H. J. A. Jensen, Chem. Phys. Lett. 137, 431 (1987).

${ }^{23}$ H. Ågren and H. J. A. Jensen, Chem. Phys. 172, 45 (1993).

${ }^{24}$ V. Carravetta, Y. Luo, and H. Ågren, Chem. Phys. 174, 141 (1993).

${ }^{25}$ V. Carravetta, H. Ågren, H. J. Å. Jensen, P. Jørgensen, and J. Olsen, J. Phys. B: At., Mol. Opt. Phys. 22, 2133 (1989).

${ }^{26}$ U. Ekström, P. Norman, V. Carravetta, and H. Ågren, Phys. Rev. Lett. 97, 143001 (2006). 
${ }^{27}$ H. Ågren, V. Carravetta, O. Vahtras, and L. G. Pettersson, Chem. Phys. Lett. 222, 75 (1994).

${ }^{28}$ U. Ekström, P. Norman, and V. Carravetta, Phys. Rev. A 73, 022501 (2006).

${ }^{29}$ U. Ekström and P. Norman, Phys. Rev. A 74, 042722 (2006).

${ }^{30}$ M. Stener, G. Fronzoni, and M. de Simone, Chem. Phys. Lett. 373, 115 (2003).

${ }^{31}$ P. Schwerdtfeger, ChemPhysChem 12, 3143 (2011).

${ }^{32}$ T. E. Meehan, K. Hermann, and F. P. Larkins, J. Phys. B: At., Mol. Opt. Phys. 28, 357 (1995).

${ }^{33}$ J. Schirmer, Phys. Rev. A 26, 2395 (1982).

${ }^{34}$ J. Schirmer, A. B. Trofimov, K. J. Randall, J. Feldhaus, A. M. Bradshaw, Y. Ma, C. T. Chen, and F. Sette, Phys. Rev. A 47, 1136 (1993).

${ }^{35}$ A. B. Trofimov, T. É. Moskovskaya, E. V. Gromov, N. M. Vitkovskaya, and J. Schirmer, J. Struct. Chem. 41, 483 (2000).

${ }^{36}$ J. Wenzel, A. Holzer, M. Wormit, and A. Dreuw, J. Chem. Phys. 142, 214104 (2015).

${ }^{37}$ M. Wormit, D. R. Rehn, P. H. Harbach, J. Wenzel, C. M. Krauter, E. Epifanovsky, and A. Dreuw, Mol. Phys. 112, 774 (2014).

${ }^{38}$ A. Dreuw and M. Wormit, Wiley Interdiscip. Rev.: Comput. Mol. Sci. 5, 82 (2015).

${ }^{39} \mathrm{M}$. Nooijen and R. J. Bartlett, J. Chem. Phys. 102, 6735 (1995).

${ }^{40}$ S. Coriani, O. Christiansen, T. Fransson, and P. Norman, Phys. Rev. A 85, 022507 (2012).

${ }^{41} \mathrm{~S}$. Coriani, T. Fransson, O. Christiansen, and P. Norman, J. Chem. Theory Comp. 8, 1616 (2012).

${ }^{42}$ S. Coriani and H. Koch, J. Chem. Phys. 143, 181103 (2015).

${ }^{43}$ M. L. Vidal, X. Feng, E. Epifanovsky, A. I. Krylov, and S. Coriani, J. Chem. Theory Comput. 15, 3117 (2019).

${ }^{44}$ P. Norman and A. Dreuw, Chem. Rev. 118, 7208 (2018).

${ }^{45}$ P. Langhoff, Chem. Phys. Lett. 22, 60 (1973).

${ }^{46}$ P. Langhoff, C. Corcoran, J. Sims, F. Weinhold, and R. Glover, Phys. Rev. A 14, 1042 (1976).

${ }^{47}$ D. L. Yeager, M. A. C. Nascimento, and V. McKoy, Phys. Rev. A 11, 1168 (1975).

${ }^{48}$ M. A. C. Nascimento and W. A. Goddard, Phys. Rev. A 16, 1559 (1977).

${ }^{49}$ M. A. C. Nascimento, J. Mol. Struct. 120, 227 (1985).

${ }^{50}$ E. Hollauer and M. A. C. Nascimento, Phys. Rev. A 42, 6608 (1990).

${ }^{51}$ H. Ågren, V. Carravetta, H. J. A. Jensen, P. Jørgensen, and J. Olsen, Phys. Rev. A 47, 3810 (1993).

${ }^{52}$ V. Carravetta, H. Ågren, D. Nordfors, and S. Svensson, Chem. Phys. Lett. 152, 190 (1988).

${ }^{53}$ R. Santra, J. Zobeley, and L. S. Cederbaum, Phys. Rev. B 64, 245104 (2001).

${ }^{54}$ K. Gokhberg, V. Vysotskiy, L. S. Cederbaum, L. Storchi, F. Tarantelli, and V. Averbukh, J. Chem. Phys. 130, 064104 (2009).

${ }^{55}$ S. Kopelke, K. Gokhberg, L. S. Cederbaum, F. Tarantelli, and V. Averbukh, J. Chem. Phys. 134, 024106 (2011).

${ }^{56}$ J. Cukras, S. Coriani, P. Decleva, O. Christiansen, and P. Norman, J. Chem. Phys. 139, 094103 (2013).

${ }^{57}$ J. Cukras, P. Decleva, and S. Coriani, J. Chem. Phys. 141, 174315 (2014).

${ }^{58}$ B. N. C. Tenorio, M. A. C. Nascimento, S. Coriani, and A. B. Rocha, J. Chem. Theory Comp. 12, 4440 (2016).

${ }^{59}$ B. N. C. Tenorio, M. A. C. Nascimento, and A. B. Rocha, J. Chem. Phys. 148, 074104 (2018).
${ }^{60}$ S. P. Neville, V. Averbukh, M. Ruberti, R. Yun, S. Patchkovskii, M. Chergui, A. Stolow, and M. S. Schuurman, J. Chem. Phys. 145, 144307 (2016).

${ }^{61}$ B. N. C. Tenorio, R. R. Oliveira, M. A. C. Nascimento, and A. B. Rocha, J. Chem. Theory Comput. 14, 5324 (2018).

${ }^{62}$ T. Helgaker, P. Jørgensen, and J. Olsen, Molecular Electronic Structure Theory (Wiley, 2004).

${ }^{63}$ H. Koch and P. Jørgensen, J. Chem. Phys. 93, 3333 (1990).

${ }^{64}$ O. Christiansen, P. Jørgensen, and C. Hättig, Int. J. Quantum Chem. 68, 1 (1998).

${ }^{65} \mathrm{O}$. Christiansen, A. Halkier, H. Koch, P. Jørgensen, and T. Helgaker, J. Chem. Phys. 108, 2801 (1998).

${ }^{66}$ L. S. Cederbaum, W. Domcke, and J. Schirmer, Phys. Rev. A 22, 206 (1980).

${ }^{67}$ F. Muller-Plathe and G. H. Diercksen, in Electronic Structure of Atoms, Molecules and Solids, Proceeding of the II Escola Brasileira de Estructure Eletronica, Olinda, Brazil, July 17-22, 1989, edited by S. Canuto, J. D’Albuquerque e Castro, and F. J. Paixao (World Scientific, Olinda, Brazil, 1990), pp. 1-29.

${ }^{68}$ J. T. Broad and W. P. Reinhardt, J. Chem. Phys. 60, 2182 (1974).

${ }^{69}$ P. Linstrom and W. Mallard, NIST Chemistry WebBook, NIST Standard Reference Database, 2017.

${ }^{70}$ K. Aidas, C. Angeli, K. L. Bak, V. Bakken, R. Bast, L. Boman, O. Christiansen, R. Cimiraglia, S. Coriani, P. Dahle, E. Dalskov, U. Ekström, T. Enevoldsen, J. J. Eriksen, P. Ettenhuber, B. Fernández, L. Ferrighi, H. Fliegl, L. Frediani, K. Hald, A. Halkier, C. Hättig, H. Heiberg, T. Helgaker, A. C. Hennum, H. Hettema, E. Hjertenaes, S. Høst, I.-M. Høyvik, M. F. Iozzi, B. Jansík, H. Jensen, D. Jonsson, P. Jørgensen, J. Kauczor, S. Kirpekar, T. Kjaergaard, W. Klopper, S. Knecht, R. Kobayashi, H. Koch, J. Kongsted, A. Krapp, K. Kristensen, A. Ligabue, O. Lutnæs, J. Melo, K. Mikkelsen, R. Myhre, C. Neiss, C. Nielsen, P. Norman, J. Olsen, J. Olsen, A. Osted, M. Packer, F. Pawlowski, T. Pedersen, P. Provasi, S. Reine, Z. Rinkevicius, T. A. Ruden, K. Ruud, V. V. Rybkin, P. Salek, C. C. M. Samson, A. S. de Merás, T. Saue, S. Sauer, B. Schimmelpfennig, K. Sneskov, A. H. Steindal, K. O. Sylvester-Hvid, P. R. Taylor, A. M. Teale, E. I. Tellgren, D. P. Tew, A. J. Thorvaldsen, L. Thøgersen, O. Vahtras, M. A. Watson, D. J. D. Wilson, M. Ziolkowski, and H. Ågren, Wiley Interdiscip. Rev.: Comput. Mol. Sci. 4, 269 (2014).

${ }^{71}$ T. H. Dunning, J. Chem. Phys. 90, 1007 (1989).

${ }^{72}$ W. J. Hehre, R. Ditchfield, and J. A. Pople, J. Chem. Phys. 56, 2257 (1972).

${ }^{73}$ K. Kaufmann, W. Baumeister, and M. Jungen, J. Phys. B: At., Mol. Opt. Phys. 22, 2223 (1989).

${ }^{74}$ A. Rohatgi, Webplotdigitizer, 2017.

${ }^{75}$ C. Amis and A. Hitchcock, Gas Phase Core Excitation Database, 2003.

${ }^{76}$ A. Hitchcock and D. Mancini, J. Electron Spectrosc. Relat. Phenom. 67, 1 (1994).

${ }^{77}$ R. McLaren, S. A. C. Clark, I. Ishii, and A. Hitchcock, Phys. Rev. A 36, 1683 (1987).

${ }^{78}$ J. Wenzel, M. Wormit, and A. Dreuw, J. Comput. Chem. 35, 1900 (2014).

${ }^{79}$ M. Tronc, G. C. King, and F. H. Read, J. Phys. B: At. Mol. Phys. 12, 137 (1979).

${ }^{80}$ I. Ishii, R. McLaren, A. P. Hitchcock, and M. B. Robin, J. Chem. Phys. 87, 4344 (1987).

${ }^{81}$ R. N. Sodhi and C. Brion, J. Electron Spectrosc. Relat. Phenom. 36, 187 (1985).

${ }^{82}$ K. C. Prince, R. Richter, M. de Simone, M. Alagia, and M. Coreno, J. Phys. Chem. A 107, 1955 (2003).

${ }^{83}$ A. Hitchcock and C. Brion, J. Electron Spectrosc. Relat. Phenom. 19, 231 (1980).

${ }^{84}$ D. C. Newbury, I. Ishii, and A. P. Hitchcock, Can. J. Chem. 64, 1145 (1986).

${ }^{85}$ D. Duflot, J. P. Flament, A. Giuliani, J. Heinesch, and M. J. Hubin-Franskin, J. Chem. Phys. 119, 8946 (2003). 